\title{
A Lottery Model for Center-Type Problems With Outliers
}

\author{
DAVID G. HARRIS, Department of Computer Science, University of Maryland \\ THOMAS PENSYL, Department of Computer Science, University of Maryland \\ ARAVIND SRINIVASAN, Department of Computer Science and Institute for Advanced \\ Computer Studies, University of Maryland \\ KHOA TRINH, Department of Computer Science, University of Maryland
}

\begin{abstract}
In this article, we give tight approximation algorithms for the $k$-center and matroid center problems with outliers. Unfairness arises naturally in this setting: certain clients could always be considered as outliers. To address this issue, we introduce a lottery model in which each client $j$ is allowed to submit a parameter $p_{j} \in[0,1]$ and we look for a random solution that covers every client $j$ with probability at least $p_{j}$. Our techniques include a randomized rounding procedure to round a point inside a matroid intersection polytope to a basis plus at most one extra item such that all marginal probabilities are preserved and such that a certain linear function of the variables does not decrease in the process with probability one.
\end{abstract}

CCS Concepts: • Theory of computation $\rightarrow$ Facility location and clustering; Rounding techniques;

Additional Key Words and Phrases: Approximation algorithms, randomized rounding

\section{ACM Reference format:}

David g. Harris, Thomas Pensyl, Aravind Srinivasan, and Khoa Trinh. 2019. A Lottery Model for Center-Type Problems With Outliers. ACM Trans. Algorithms 15, 3, Article 36 (June 2019), 25 pages.

https://doi.org/10.1145/3311953

\section{INTRODUCTION}

The classic $k$-center and Knapsack Center problems are known to be approximable to within factors of 2 and 3 , respectively [8]. These results are best possible unless $\mathrm{P}=\mathrm{NP}[8,9]$. In these problems, we are given a metric graph $G$ and want to find a subset $\mathcal{S}$ of vertices of $G$ subject to either a cardinality constraint or a knapsack constraint such that the maximum distance from any vertex to the nearest vertex in $\mathcal{S}$ is as small as possible. We shall refer to vertices in $G$ as clients. Vertices in $\mathcal{S}$ are also called centers.

Research supported in part by NSF Awards CNS-1010789 and CCF-1422569, and by a research award from Adobe Inc. and Google LLC. A preliminary version of this work appeared in the Proc. International Workshop on Approximation Algorithms for Combinatorial Optimization Problems (APPROX), 2017.

Authors' addresses: D. G. Harris, Thomas Pensyl, Department of Computer Science, University of Maryland, College Park, MD, 20742, USA; emails: davidgharris29@gmail.com, tpensyl@cs.umd.edu; A. Srinivasan, Department of Computer Science and Institute for Advanced Computer Studies, University of Maryland, College Park, MD, 20742, USA; email: srin@cs.umd.edu; K. Trinh, Department of Computer Science, University of Maryland, College Park, MD, 20742, USA; email:khoa@cs.umd.edu.

Authors Current address: T. Pensyl, Bandwith Inc., Raleigh, NC, USA; email: tpensyl@bandwidth.com; K. Trinh, Google LLC, Mountain View, CA, USA; email: khoatrinh@google.com.

Permission to make digital or hard copies of all or part of this work for personal or classroom use is granted without fee provided that copies are not made or distributed for profit or commercial advantage and that copies bear this notice and the full citation on the first page. Copyrights for components of this work owned by others than ACM must be honored. Abstracting with credit is permitted. To copy otherwise, or republish, to post on servers or to redistribute to lists, requires prior specific permission and/or a fee. Request permissions from permissions@acm.org.

(C) 2019 Association for Computing Machinery.

1549-6325/2019/06-ART36 \$15.00

https://doi.org/10.1145/3311953 
It is not difficult to see that a few outliers (i.e., very distant clients) may result in a very large optimal radius in the center-type problems. This issue was raised by Charikar et al. [4], who proposed a robust model in which we are given a parameter $t$ and only need to serve $t$ out of given $n$ clients (i.e., $n-t$ outliers may be ignored in the solution). Here we consider three robust center-type problems: the Robust $k$-Center (RkCenter) problem, the Robust Knapsack Center (RKnapCenter) problem, and the Robust Matroid Center (RMatCenter) problem.

Formally, an instance $\mathcal{I}$ of the RkCenter problem consists of a set $V$ of vertices, a metric distance $d$ on $V$, an integer $k$, and an integer $t$. Let $n=|V|$ denote the number of vertices (clients). The goal is to choose a set $\mathcal{S} \subseteq V$ of centers (facilities) such that (i) $|\mathcal{S}| \leq k$, (ii) there is a set of covered vertices (clients) $C \subseteq V$ of size at least $t$, and (iii) the objective function

$$
R:=\max _{j \in C} \min _{i \in \mathcal{S}} d(i, j)
$$

is minimized.

In the RKnapCenter problem, each vertex $i \in V$ has a weight $w_{i} \in[0,1]$, and the cardinality constraint (i) is replaced by the knapsack constraint: $\sum_{i \in \mathcal{S}} w_{i} \leq 1$. Similarly, in the RMatCenter problem, the constraint (i) is replaced by a matroid constraint: $\mathcal{S}$ must be an independent set of a given matroid $\mathcal{M}$. Here we assume that we have access to the rank oracle of $\mathcal{M}$.

In [4], Charikar et al. introduced a greedy algorithm for the RkCenter problem that achieves an approximation ratio of 3. Recently, Chakrabarty et al. [3] give a 2-approximation algorithm for this problem. Since the $k$-center problem is a special case of the RkCenter problem, this ratio is best possible unless $\mathrm{P}=\mathrm{NP}$.

The RKnapCenter problem was first studied by Chen et al. [5], which showed that one can achieve an approximation ratio of 3 if allowed to slightly violate the knapsack constraint by a factor of $(1+\epsilon)$. It is still unknown whether there exists a true approximation algorithm for this problem. The current inapproximability bound is still 3 due to the hardness of the Knapsack Center problem. The current best approximation guarantee for the RMatCenter problem is 7 by Chen et al. [5]. This problem has a hardness of $(3-\epsilon)$ via a reduction from the $k$-supplier problem.

From a practical viewpoint, unfairness arises inevitably in the robust model: some clients might always be considered as outliers and hence not covered within the guaranteed radius. To address this issue, we introduce a lottery model for these problems. The idea is to randomly pick a solution from a public list such that each client $j \in V$ is guaranteed to be covered with probability at least $p_{j}$, where $p_{j} \in[0,1]$ is the success rate requested by $j$. This is also motivated by the fact that different clients may have different tolerances of getting connected to their closest facility. One possible way of determining the $p_{j}$ values is to let each client $j$ pay for the chance of being served. Note that the robust model is a special case when $p_{j}=0$ for all $j \in V$. Similarly, when all $p_{j}$ 's are equal to 1 , it becomes the standard model where all clients must be connected.

The lottery model might also be useful in the context of clustering. Recall that clustering is a fundamental task in unsupervised machine learning. Basically, we want to partition a set of data points to clusters in such a way that the points in the same cluster are "similar" to each other. The $k$-center clustering is one of the popular approaches to this task. (See also $k$-means clustering [1, 10] and $k$-median clustering $[2,13,16]$.) Naturally, the robust model can be applied to get rid of some "bad data points" or "noise" and hence improve the overall quality of all clusters. Here overfitting may occur when this model misclassifies some good points as outliers. The lottery model offers the flexibility to decide whether a point should be included in the solution via the $p_{j}$ values.

In this article, we introduce new approximation algorithms for these robust center problems under the lottery model. (Note that this model has been used recently for the Knapsack Center problems [7], although the techniques and problems in that paper are different from ours.) We also propose improved approximation algorithms for the RkCenter problem and the RMatCenter problem. 


\subsection{The Lottery Model}

In this section, we formally define our lottery model for the above-mentioned problems. First, the Fair Robust $k$-Center (FRkCenter) problem is formulated as follows. Besides the parameters $V, d, k$, and $t$, each vertex $j \in V$ has a "target" probability $p_{j} \in[0,1]$. We are interested in the minimum radius $R$ for which there exists a distribution $\mathcal{D}$ on subsets of $V$ such that a set $\mathcal{S}$ drawn from $\mathcal{D}$ satisfies the following constraints:

Coverage constraint: $|C| \geq t$ with probability one, where $C$ is the set of all clients in $V$ that are within radius $R$ from some center $\mathcal{S}$.

Fairness constraint: $\operatorname{Pr}[j \in C] \geq p_{j}$ for all $j \in V$, where $C$ is as in the coverage constraint.

Cardinality constraint: $|\mathcal{S}| \leq k$ with probability one.

We shall refer to such a minimum radius $R$ as the optimal radius of the instance. Here we aim for a polynomial-time, randomized algorithm that can sample from $\mathcal{D}$. Note that the RkCenter is a special of this variant in which all $p_{j}$ 's are set to be zero.

The Fair Robust Knapsack Center (FRKnapCenter) problem and Fair Robust Matroid Center (FRMatCenter) problem are defined similarly except that we replace the cardinality constraint by a knapsack constraint and a matroid constraint, respectively. More formally, in the FRKnapCenter problem, each vertex $i$ has a weight $w_{i} \in[0,1]$, and we require the total weight of centers in $\mathcal{S}$ to be at most 1 with probability one. Similarly, in the FRMatCenter problem, we are given a matroid $\mathcal{M}$ and we require the solution $\mathcal{S}$ to be an independent set of $\mathcal{M}$ with probability one.

\subsection{Our Contributions and Techniques}

First of all, we give tight approximation algorithms for the RkCenter and RMatCenter problems.

THEOREm 1.1. There exist a 2-approximation algorithm for the RkCenter problem ${ }^{1}$ and a 3approximation algorithm for the RMatCenter problem.

Our main results for the lottery model are summarized in the following theorems.

THEOREM 1.2. For any given constant $\epsilon>0$ and any instance $\mathcal{I}=(V, d, k, t, \vec{p})$ of the FRkCenter problem, there is a randomized polynomial-time algorithm $\mathcal{A}$ which can compute a random solution $\mathcal{S}$ such that

$-|\mathcal{S}| \leq k$ with probability one,

$-|C| \geq(1-\epsilon) t$, where $C$ is the set of all clients within radius $2 R$ from some center in $\mathcal{S}$ and $R$ is the optimal radius, and

$-\operatorname{Pr}[j \in C] \geq(1-\epsilon) p_{j}$ for all $j \in V$.

TheOREM 1.3. For any $\epsilon>0$ and any instance $\mathcal{I}=(V, d, w, t, \vec{p})$ of the FRKnapCenter problem, there is a randomized polynomial-time algorithm $\mathcal{A}$ which can return random solution $\mathcal{S}$ such that

$-\sum_{i \in \mathcal{S}} w_{i} \leq 1+\epsilon$ with probability one,

$-|C| \geq t$, where $C$ is the set of vertices within distance $3 R$ of $\mathcal{S}$, and

$-\operatorname{Pr}[j \in C] \geq p_{j}$ for all $j \in V$.

Finally, the FRMatCenter can be reduced to (randomly) rounding a point in a matroid intersection polytope. We design a randomized rounding algorithm which can output a pseudo solution, which consists of a basis plus one extra center. By using a preprocessing step and a configuration

\footnotetext{
${ }^{1}$ A 2-approximation algorithm has also been found independently by Chakrabarty et al. [3], and in a private discussion between Marek Cygan and Samir Khuller. Our algorithm here is different from the algorithm in [3].
} 
LP, we can satisfy the matroid constraint exactly (knapsack constraint, respectively) while slightly violating the coverage and fairness constraints in the FRMatCenter (FRKnapCenter, respectively) problem. We believe these techniques could be useful in other facility-location problems (e.g., the matroid median problem $[11,15])$ as well.

THEOREM 1.4. For any given constant $\gamma>0$ and any instance $\mathcal{I}=(V, d, \mathcal{M}, t, \vec{p})$ of the FRMatCenter (FRKnapCenter, respectively) problem, there is a randomized polynomial-time algorithm $\mathcal{A}$ which can return a random solution $\mathcal{S}$ such that

$-\mathcal{S}$ is an independent set of $\mathcal{M}$ with probability one, $(w(\mathcal{S}) \leq 1$ with probability one, respectively),

$-|C| \geq t-\gamma^{2} n$, where $C$ is the set of vertices within distance $3 R$ of $\mathcal{S}$, and

-there exists a set $T \subseteq V$ of size at least $(1-\gamma) n$, which does not depend on the randomness of $\mathcal{A}$, such that $\operatorname{Pr}[j \in C] \geq p_{j}-\gamma$ for all $j \in T$.

\subsection{Organization}

The rest of this article is organized as follows. In Section 2, we review some basic properties of matroids and discuss a filtering algorithm which is used in later algorithms. Then we develop approximation algorithms for the FRkCenter, FRKnapCenter, and FRMatCenter problems in the next three sections.

\section{PRELIMINARIES}

All algorithms in this article are based on rounding an LP solution. In general, for each vertex $i \in V$, we have a variable $y_{i} \in[0,1]$ which represents the probability that we want to pick $i$ in our solution. (In the standard model, $y_{i}$ is the "extent" that $i$ is opened.) In addition, for each pair of $i, j \in V$, we have a variable $x_{i j} \in[0,1]$ which represents the probability that $j$ is connected to $i$.

Note that in all center-type problems, the optimal radius $R$ is always the distance between two vertices. Therefore, we can "guess" the value of $R$ in $O(\log n)$ time by performing a binary search over the $n^{2}$ possible distances in matrix $d$. More precisely, for a guess value of $R$, we will try to solve for a solution $(x, y)$ which satisfies a set of linear constraints (to be specified) that depend on $R$. If such a solution does not exist, our guess radius is too low and we need to try larger values. Otherwise, we shall try smaller ones. The process will take at most $O(\log n)$ iterations and yield a lower-bound $R^{*}$ of the optimal radius. For the ease of notation (and WLOG), assume that we obtain the exact value of the optimal radius $R$.

For any $j \in V$, we define $B_{j}:=\{i \in V: d(i, j) \leq R\}$. Depending on a specific problem, we may have different constraints on $x_{i j}$ 's and $y_{i}$ 's. In general, the following constraints are valid in most of the problems here:

$$
\begin{aligned}
\sum_{j \in V} \sum_{i \in B_{j}} x_{i j} & \geq t, \\
\sum_{i \in B_{j}} x_{i j} & \leq 1, \quad \forall j \in V, \\
x_{i j} & \leq y_{i}, \quad \forall i, j \in V, \\
y_{i}, x_{i j} & \geq 0, \quad \forall i, j \in V .
\end{aligned}
$$

For the fair variants, we may also require that

$$
\sum_{i \in B_{j}} x_{i j} \geq p_{j}, \quad \forall j \in V .
$$


Constraint (1) says that at least $t$ vertices should be covered. Constraint (2) ensures that each vertex is only connected to at most one center. Constraint (3) means vertex $j$ can only connect to center $i$ if it is open. Constraint (5) says that the total probability of $j$ being connected should be at least $p_{j}$.

Suppose $(x, y)$ is a solution satisfying all the required constraints. We let $F_{j}:=\{i \in V: d(i, j) \leq$ $\left.R \wedge x_{i j}>0\right\}$ and $s_{j}:=\sum_{i \in B_{j}} x_{i j}$. We shall refer to $F_{j}$ as a cluster with cluster center $j$. Essentially, $F_{j}$ is the set of vertices that can be connected to $j$ if it is chosen in the solution set $\mathcal{S}$. (Note that it could be that $j \notin F_{j}$.) The first step of all algorithms in this article is to use the following filtering algorithm to obtain a maximal collection of disjoint clusters. The algorithm will return the set $V^{\prime}$ of cluster centers of the chosen clusters. In the process, we also keep track of the number $c_{j}$ of other clusters removed by $F_{j}$ for each $j \in V^{\prime}$.

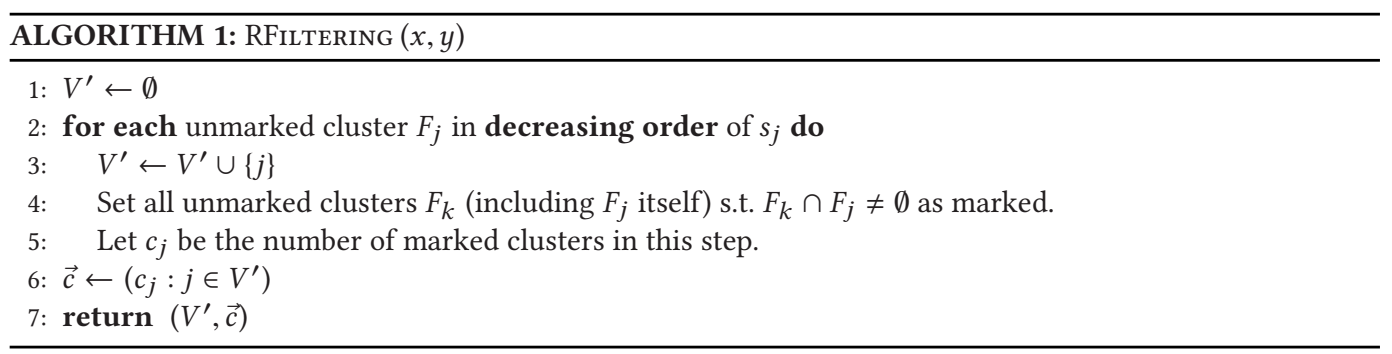

\section{THE $k$-CENTER PROBLEMS WITH OUTLIERS}

In this section, we first give a simple 2-approximation algorithm for the RkCenter problem. Then, we give an approximation algorithm for the FRkCenter problem, proving Theorem 1.2.

\subsection{The Robust $k$-Center Problem}

Suppose $\mathcal{I}=(V, d, k, t)$ is an instance of the RkCenter problem with the optimal radius $R$. Consider the polytope $\mathcal{P}_{\mathrm{RkCenter}}$ containing points $(x, y)$ satisfying constraints (1)-(4), and the cardinality constraint:

$$
\sum_{i \in V} y_{i} \leq k
$$

Since $R$ is the optimal radius, it is not difficult to check that $\mathcal{P}_{\mathrm{RkCenter}} \neq \emptyset$. Let us pick any fractional solution $(x, y) \in \mathcal{P}_{\mathrm{RkCenter}}$. The next step is to round $(x, y)$ into an integral solution using the following simple algorithm.

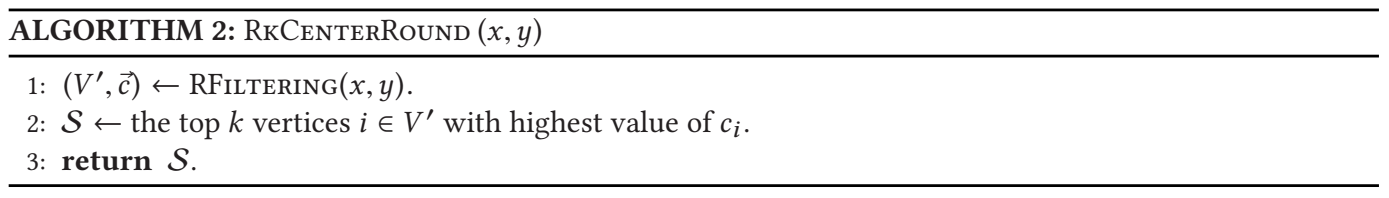

Analysis. By construction, the algorithm returns a set $\mathcal{S}$ of at most $k$ open centers. Note that, for each $i \in \mathcal{S}, c_{i}$ is the number of distinct clients within radius $2 R$ from $i$. Thus, it suffices to show that $\sum_{i \in \mathcal{S}} c_{i} \geq t$. By inequality (2), we have $s_{j} \leq 1$ for all $j \in V^{\prime}$. Thus,

$$
\sum_{i \in V^{\prime}} c_{i} s_{i} \geq \sum_{i \in V} s_{i} \geq t
$$


where the first inequality is due to the greedy choice of vertices in $V^{\prime}$ and the second inequality follows from (1). Now recall that the clusters whose centers in $V^{\prime}$ are pairwise disjoint. By constraint (6),

$$
\sum_{i \in V^{\prime}} s_{i} \leq \sum_{i \in V^{\prime}} y\left(F_{i}\right) \leq \sum_{i \in V} y_{i} \leq k
$$

It follows by the choice of $\mathcal{S}$ that $\sum_{i \in \mathcal{S}} c_{i} \geq t$. This concludes the first part of Theorem 1.1.

\subsection{The Fair Robust $k$-Center Problem}

Let $\mathcal{I}=(V, d, k, t, \vec{p})$ be an instance of the FRkCenter problem with the optimal radius $R$. Fix any $\epsilon>0$. If $k \leq 2 / \epsilon$, then we can generate all possible $O\left(n^{1 / \epsilon}\right)$ solutions and then solve an LP to obtain the corresponding marginal probabilities. So the problem can be solved easily in this case. We will assume that $k \geq 2 / \epsilon$ for the rest of this section. Consider the polytope $\mathcal{P}_{\text {FRkCenter }}$ containing points $(x, y)$ satisfying constraints (1)-(4), the fairness constraint (5), and the cardinality constraint (6). We now show that $\mathcal{P}_{\text {FRkCenter }}$ is actually a valid relaxation polytope.

\section{Proposition 3.1. The polytope $\mathcal{P}_{\text {FRkCenter }}$ is non-empty.}

Proof. It suffices to point out a solution $(x, y) \in \mathcal{P}_{\text {FRkCenter }}$. Since $R$ is the optimal radius, there exists a distribution $\mathcal{D}$ satisfying the coverage, fairness, and cardinality constraints. Suppose $\mathcal{S}$ is sampled from $\mathcal{D}$ and $C$ is the set of all clients in $V$ that are within radius $R$ from some center $\mathcal{S}$. We now set $y_{i}:=\operatorname{Pr}[i \in \mathcal{S}]$ for all $i \in V$. Since $|\mathcal{S}| \leq k$ with probability one, we have $\sum_{i \in V} y_{i}=$ $\mathrm{E}[|\mathcal{S}|] \leq k$, and hence constraint (6) is valid.

We construct the assignment variable $x$ as follows. For each $j \in V$, set $z_{j}:=0$. Then for each $i \in B_{j}$, set $x_{i j}:=\min \left\{y_{i}, 1-z_{j}\right\}$ and update $z_{j}:=z_{j}+x_{i j}$. It is not hard to see that inequalities (2) and (3) hold by this construction. Now let us fix any $j \in V$. By fairness guarantee of $\mathcal{D}$ and the union bound, we have

$$
p_{j} \leq \operatorname{Pr}[j \in C] \leq \sum_{i \in B_{j}} y_{i} .
$$

Thus, by construction of $x$, we have

$$
\sum_{i \in B_{j}} x_{i j} \geq \operatorname{Pr}[j \in C] \geq p_{j},
$$

and hence inequality (5) is satisfied. Finally, we have

$$
\mathrm{E}[|C|]=\sum_{j \in V} \operatorname{Pr}[j \in C] \leq \sum_{j \in V} \sum_{i \in B_{j}} x_{i j} .
$$

Since $|C| \geq t$ with probability one, $\mathrm{E}[|C|] \geq t$, implying that inequality (1) holds.

Our algorithm is as follows.

Analysis. First, note that one can find such a vector $\delta$ in line 4 as the system of $\delta\left(V^{\prime}\right)=0$ and $\vec{c} \cdot \delta=0$ consists of two constraints and at least three variables (and hence is underdetermined.) Thus, the algorithm rounds at least one fractional variable per iteration, and terminates after $O(n)$ rounds. Let $\mathcal{S}$ denote the (random) solution returned by FRKCENTERRound and $C$ be the set of all clients within radius $3 R$ from some center in $\mathcal{S}$. Theorem 1.2 can be verified by the following propositions.

Proposition 3.2. $|\mathcal{S}| \leq k$ with probability one. 


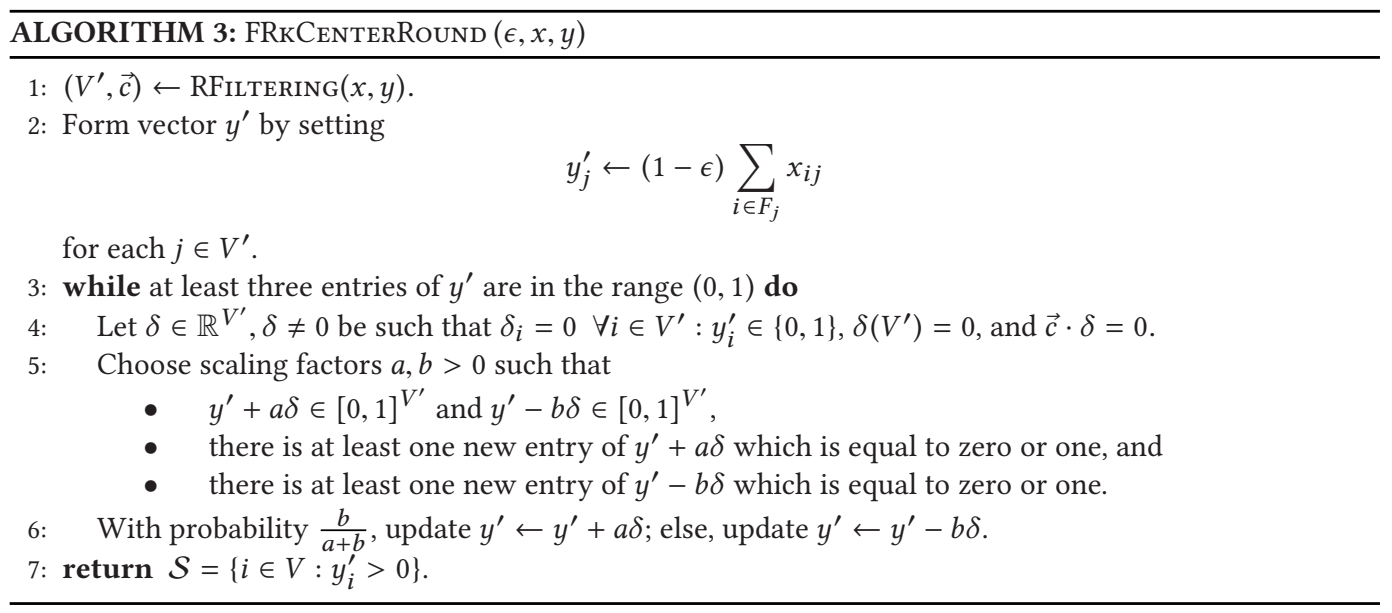

Proof. By definition of $y^{\prime}$ at line 2 of FRkCenterRound, we have

$$
y^{\prime}\left(V^{\prime}\right)=(1-\epsilon) \sum_{j \in V^{\prime}} \sum_{i \in F_{j}} x_{i j} \leq(1-\epsilon) \sum_{j \in V^{\prime}} \sum_{i \in F_{j}} y_{i} \leq(1-\epsilon) k \leq k-2,
$$

since $k \geq 2 / \epsilon$. Note that the sum $y^{\prime}\left(V^{\prime}\right)$ is never changed in the while loop (lines $4 \ldots 7$ ) because $\delta\left(V^{\prime}\right)=0$. Then the final vector $y^{\prime}$ contains at most two fractional values at the end of the while loop. By rounding these two values to one, the size of $\mathcal{S}$ is indeed at most $k$.

Proposition 3.3. $|C| \geq(1-\epsilon) t$ with probability one.

Proof. At the beginning of the while loop, we have

$$
\vec{c} \cdot y^{\prime}=\sum_{j \in V^{\prime}} c_{j} y_{j}^{\prime}\left(F_{j}\right)=(1-\epsilon) \sum_{j \in V^{\prime}} c_{j} s_{j} \geq(1-\epsilon) \sum_{j \in V} s_{j} \geq(1-\epsilon) t
$$

Again, the quantity $\vec{c} \cdot y^{\prime}$ is unchanged in the while loop because $\vec{c} \cdot \delta=0$ implies that $\vec{c} \cdot\left(y^{\prime}+\right.$ $a \delta)=\vec{c} \cdot y^{\prime}$ and $\vec{c} \cdot\left(y^{\prime}-b \delta\right)=\vec{c} \cdot y^{\prime}$ in each iteration. Note that if $y^{\prime} \in\{0,1\}^{V^{\prime}}$, then $\vec{c} \cdot y^{\prime}$ is the number of clients within radius $2 R$ from some center $i$ such that $y_{i}^{\prime}=1$. Basically, we round the two remaining fractional values of $y^{\prime}$ to one in line 8 ; and hence, the dot product should be still at least $(1-\epsilon) t$.

Proposition 3.4. $\operatorname{Pr}[j \in C] \geq(1-\epsilon) p_{j}$ for all $j \in V$.

Proof. Fix any $j \in V$. The algorithm RFiltering guarantees that there exists $k \in V^{\prime}$ such that $F_{j} \cap F_{k} \neq \emptyset$ and $s_{k} \geq s_{j}$. Let $Y$ denote the final vector $y^{\prime}$ at line 7 . We also refer to $y^{\prime}$ as the original vector defined at line 2 in this proof. Now we claim that $\mathrm{E}\left[Y_{k}\right]=y_{k}^{\prime}$. This is because the expected value of $y_{k}^{\prime}$ does not change after any single iteration. Let $y^{(i)}$ and $y^{(i+1)}$ denote the value of $y_{k}^{\prime}$ after the $i$-th and $(i+1)$-th iteration, respectively. After any $i$ iterations, the expectation of $y^{(i+1)}$ conditioned on $y^{(i)}$ is

$$
\mathrm{E}\left[y^{(i+1)}\right]=\left(y^{(i)}+a \delta\right) \frac{b}{a+b}+\left(y^{(i)}-b \delta\right) \frac{a}{a+b}=y^{(i)} .
$$

It follows by a simple induction that $\mathrm{E}\left[Y_{k}\right]=y_{k}^{\prime}$. Then

$$
\operatorname{Pr}[k \in \mathcal{S}]=\operatorname{Pr}\left[Y_{k}>0\right] \geq \operatorname{Pr}\left[Y_{k}=1\right]=\mathrm{E}\left[Y_{k}\right]=y_{k}^{\prime}=(1-\epsilon) s_{k} .
$$


Therefore,

$$
\operatorname{Pr}[j \in C] \geq \operatorname{Pr}[k \in \mathcal{S}] \geq(1-\epsilon) s_{k} \geq(1-\epsilon) s_{j} \geq(1-\epsilon) p_{j},
$$

by constraint (5).

\section{THE KNAPSACK CENTER PROBLEMS WITH OUTLIERS}

We study the RKnapCenter and FRKnapCenter problems in this section. Recall that in these problems, each vertex has a weight and we want to make sure that the total weight of the chosen centers does not exceed 1. We first give a 3-approximation algorithm for the RKnapCenter problem that slightly violates the knapsack constraint. Although this is not better than the known result by [5], both our algorithm and analysis here are more natural and simpler. It serves as a starting point for the next results. For the FRKnapCenter, we show that it is possible to satisfy the knapsack constraint exactly with small violations in the coverage and fairness constraints.

\subsection{The Robust Knapsack Center Problem}

Suppose $\mathcal{I}=(V, d, w, t)$ is an instance of the RKnapCenter problem with the optimal radius $R$. Consider the polytope $\mathcal{P}_{\text {RKnapCenter }}$ containing points $(x, y)$ satisfying constraints (1)-(4), and the knapsack constraint:

$$
\sum_{i \in V} w_{i} y_{i} \leq 1
$$

Again, it is not difficult to check that $\mathcal{P}_{\text {RKnapCenter }} \neq \emptyset$. Let us pick any fractional solution $(x, y) \in$ $\mathcal{P}_{\text {RKnapCenter. }}$ Our pseudo-approximation algorithm to round $(x, y)$ is as follows.

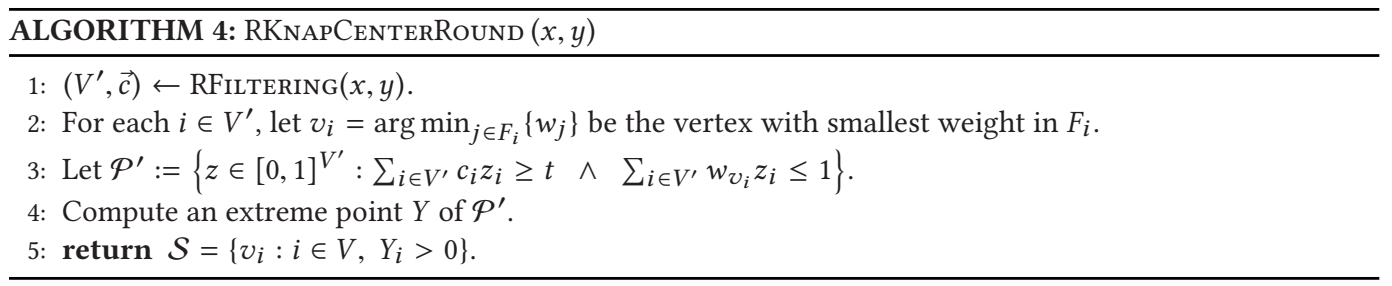

Analysis. We first claim that $\mathcal{P}^{\prime} \neq \emptyset$ which implies that the extreme point $Y$ of $\mathcal{P}^{\prime}$ (in line 4) does exist. To see this, we claim that the vector $s$ lies in $\mathcal{P}^{\prime}$. We have

$$
\sum_{i \in V^{\prime}} c_{i} s_{i} \geq \sum_{i \in V} s_{i} \geq t
$$

Also,

$$
\sum_{i \in V^{\prime}} w_{v_{i}} s_{i}=\sum_{i \in V^{\prime}} w_{v_{i}} \sum_{j \in F_{i}} x_{j i} \leq \sum_{i \in V^{\prime}} w_{v_{i}} \sum_{j \in F_{i}} y_{j} \leq \sum_{i \in V^{\prime}} \sum_{j \in F_{i}} w_{j} y_{j} \leq \sum_{i \in V} w_{i} y_{i} \leq 1
$$

All the inequalities follow from LP constraints and definitions of $s_{i}, c_{i}$, and $v_{i}$.

PROPOSITION 4.1. The solution $\mathcal{S}$ returned by RKNAPCENTERROUND satisfies $w(\mathcal{S}) \leq 1+2 w_{\text {max }}$ and $|C| \geq t$, where $C$ is the set of vertices within distance $3 R$ of $\mathcal{S}$ and $w$ max is the maximum weight of any vertex in $V$.

Proof. First, observe that any extreme point of $\mathcal{P}^{\prime}$ has at most two fractional values. (In the worst case, an extreme point $z$ is fully determined by $\left|V^{\prime}\right|-2$ tight constraints of the form $z_{i}=0$ or $z_{i}=1, \sum_{i \in V^{\prime}} c_{i} z_{i}=t$, and $\sum_{i \in V^{\prime}} w_{v_{i}} z_{i}=1$.) By construction of $\mathcal{S}$, there are at most two vertices $i^{*}, i^{* *}$ such that $Y_{i^{*}}, Y_{i^{* *}}$ are fractional. Thus, 


$$
w(\mathcal{S})=\sum_{i \in \mathcal{S} \backslash\left\{i^{*}, i^{* *}\right\}} w_{v_{i}} Y_{i}+w_{i^{*}}+w_{i^{* *}} \leq 1+2 w_{\max } .
$$

Recall that each $i \in V^{\prime}$ has $c_{i}$ clients within distance $2 R$ (and each client is counted only one time). By the triangle inequality, these clients are within distance $3 R$ from $v_{i}$. Thus, $\mathcal{S}$ covers at least

$$
\sum_{i \in \mathcal{S} \backslash\left\{i^{*}, i^{* *}\right\}} c_{i} Y_{i}+c_{i^{*}}+c_{i^{* *}} \geq \sum_{i \in \mathcal{S}} c_{i} Y_{i} \geq t
$$

clients within radius $3 R$.

\subsection{The Fair Robust Knapsack Center Problem}

In this section, we will first consider a simple algorithm that only violates the knapsack constraint by two times the maximum weight of any vertex. Then using a configuration polytope to "condition" on the set of "big" vertices, we show that it is possible to either violate the budget by $(1+\epsilon)$ or to preserve the knapsack constraint while slightly violating the coverage and fairness constraints.

4.2.1 Basic Algorithm. Suppose $\mathcal{I}=(V, d, w, t, \vec{p})$ is an instance of the FRKnapCenter problem with the optimal radius $R$. Consider the polytope $\mathcal{P}_{\text {FRKnapCenter }}$ containing points $(x, y)$ satisfying constraints (1)-(4), the fairness constraint (5), and the knapsack constraint (7). The proof that $\mathcal{P}_{\text {FRKnapCenter }} \neq \emptyset$ is very similar to that of Proposition 3.1 and is omitted here.

The following algorithm is a randomized version of RKNAPCENTERRound.

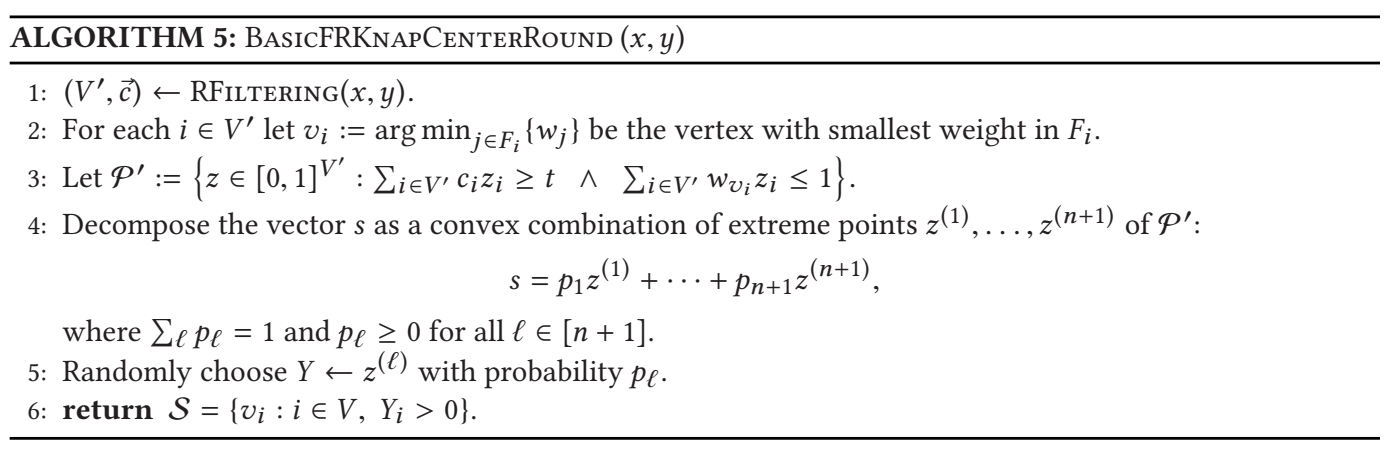

Note that $\mathcal{P}^{\prime} \neq \emptyset$, and so the decomposition at line 4 is well-defined (see the analysis in Section 4.1).

Proposition 4.2. The algorithm BASICFRKNAPCENTERROUND returns a random solution $\mathcal{S}$ such that $w(\mathcal{S}) \leq 1+2 w_{\max },|C| \geq t$, and $\operatorname{Pr}[j \in C] \geq p_{j}$ for all $j \in V$, where $C$ is the set of vertices within distance $3 R$ of $\mathcal{S}$ and $w$ max is the maximum weight of any vertex in $V$.

Proof. By similar arguments to the proof of Proposition 4.1, we have $w(\mathcal{S}) \leq 1+2 w_{\max }$ and $|C| \geq t$. To obtain the fairness guarantee, observe that $v_{i} \in \mathcal{S}$ with probability at least $z_{i}=s_{i}$. For any $j \in V$, let $k \in V^{\prime}$ be the vertex that removed $j$ in the filtering step. We have

$$
\operatorname{Pr}[j \in C] \geq \operatorname{Pr}\left[v_{k} \in \mathcal{S}\right] \geq s_{k} \geq s_{j} \geq p_{j},
$$

where the penultimate inequality is due to our greedy choice of $k$ in RFILTERING.

4.2.2 An Algorithm Slightly Violating the Budget Constraint. Fix a small parameter $\epsilon>0$. A vertex $i$ is said to be big iff $w_{i}>\epsilon$. Let $\mathcal{U}$ denote the collection of all feasible sets of big vertices. That is, the weight of every set $U \in \mathcal{U}$ should be at most 1 . Since a solution can contain at most 
$1 / \epsilon$ big vertices, we have $|\mathcal{U}| \leq n^{O(1 / \epsilon)}$. Next, for each set $U \in \mathcal{U}$, we introduce a new variable $q_{U}$ which represents the probability that, when sampling a solution $\mathcal{S}$ from the optimal distribution, $U$ is the exact set of all big vertices in $\mathcal{S}$. Consider the configuration polytope $\mathcal{P}_{\text {config } 1}$ containing points $(x, y, q)$ with the following constraints:

$$
\begin{cases}\sum_{U \in \mathcal{U}} q_{U}=1, & \\ \sum_{i \in B_{j}} x_{i j}^{U} \leq q_{U}, & \forall j \in V, U \in \mathcal{U} \\ \sum_{U \in \mathcal{U}} \sum_{i \in B_{j}} x_{i j}^{U} \geq p_{j}, & \forall j \in V \\ x_{i j}^{U} \leq y_{i}^{U}, & \forall i, j \in V, U \in \mathcal{U} \\ \sum_{i \in V} w_{i} y_{i}^{U} \leq q_{U}, & \forall U \in \mathcal{U} \\ \sum_{j \in V} \sum_{i \in B_{j}} x_{i j}^{U} \geq q_{U} t, & \\ y_{i}^{U}=1, & \forall U \in \mathcal{U}, i \in U \\ y_{i}^{U}=0, & \forall U \in \mathcal{U}, i \in V \backslash U, w_{i}>1 / \epsilon \\ x_{i j}^{U}, y_{i}^{U}, q_{U} \geq 0, & \forall i, j \in V, U \in \mathcal{U} .\end{cases}
$$

We first claim that $\mathcal{P}_{\text {config } 1}$ is a valid relaxation polytope for the problem.

Proposition 4.3. The polytope $\mathcal{P}_{\text {config1 }}$ is non-empty.

Proof. Fix any optimal distribution $\mathcal{D}$. Suppose $\mathcal{S}$ is sampled from $\mathcal{D}$. For any $U \in \mathcal{U}$, let $\mathcal{E}(U)$ be the event that $U \subseteq \mathcal{S}$ and $\mathcal{S} \backslash U$ contains no big vertex, and let $q_{U}=\operatorname{Pr}[\mathcal{E}(U)]$. It is clear that $\sum_{U \in \mathcal{U}} q_{U}=1$. Let $x_{i j}^{U}=\operatorname{Pr}[\mathcal{E}(U) \wedge j$ is connected to $i]$ and let $y_{i}^{U}=\operatorname{Pr}[\mathcal{E}(U) \wedge i \in \mathcal{S}]$.

Now observe that

$$
q_{U}=\operatorname{Pr}[\mathcal{E}(U)]=\sum_{i \in B_{j}} \operatorname{Pr}[j \text { is connected to } i \wedge \mathcal{E}(U)]=\sum_{i \in B_{j}} x_{i j}^{U} .
$$

Similarly,

$$
\begin{aligned}
p_{j} & \leq \operatorname{Pr}[j \text { is connected }]=\sum_{U \in \mathcal{U}} \operatorname{Pr}[j \text { is connected } \wedge \mathcal{E}(U)] \\
& =\sum_{U \in \mathcal{U}} \sum_{i \in B_{j}} \operatorname{Pr}[j \text { is connected to } i \wedge \mathcal{E}(U)]=\sum_{U \in \mathcal{U}} \sum_{i \in B_{j}} x_{i j}^{U} .
\end{aligned}
$$

Note that $x_{i j}^{U} / q_{U}$ and $y_{i}^{U} / q_{U}$ are the probabilities that $j$ is connected to $i$ and $i \in \mathcal{S}$ conditioned on $\mathcal{E}(U)$, respectively. Since the number of connected clients is at least $t$ with probability one, we have

$$
t \geq \mathrm{E}[\# \text { connected clients } \mid \mathcal{E}(U)]=\sum_{j \in V} \sum_{i \in B_{j}} \operatorname{Pr}[j \text { is connected to } i \mid \mathcal{E}(U)]=\sum_{j \in V} \sum_{i \in B_{j}} x_{i j}^{U} / q_{U} .
$$

Similarly, $w(\mathcal{S}) \leq 1$ with probability one and so

$$
1 \geq \mathrm{E}[w(\mathcal{S}) \mid U]=\sum_{i \in V} w_{i}\left(y_{i}^{U} / q_{U}\right)
$$

The other constraints can be verified easily. We conclude that $(x, y, q) \in \mathcal{P}_{\text {config1 }}$.

We use the following Algorithm 6 to round any $(x, y, q) \in \mathcal{P}_{\text {config } 1}$ :

We are now ready to prove Theorem 1.3.

Proof of Theorem 1.3. We will show that solution $\mathcal{S}$ returned by FRKnapCenterRound 1 satisfies the requirements of Theorem 1.3. Let $\mathcal{E}(U)$ denote the event that it selects $U \in \mathcal{U}$. Note 


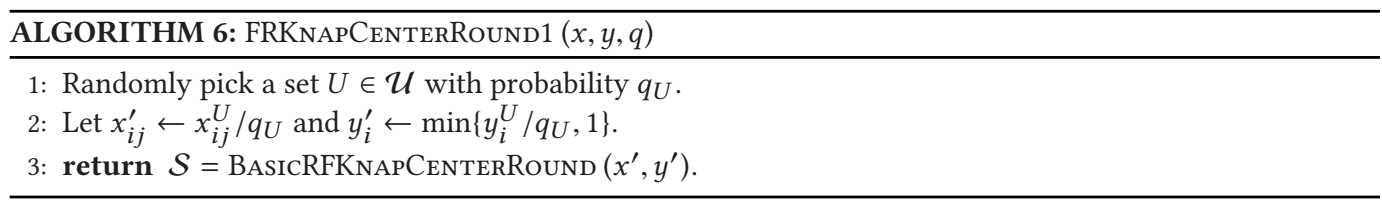

that $\left(x^{\prime}, y^{\prime}\right)$ satisfies the following constraints:

$$
\begin{aligned}
\sum_{j \in V} \sum_{i \in B_{j}} x_{i j}^{\prime} & \geq t, \\
\sum_{i \in B_{j}} x_{i j}^{\prime} & \leq 1, \quad \forall j \in V, \\
\sum_{i \in B_{j}} x_{i j}^{\prime} & =\sum_{i \in B_{j}} x_{i j} / q_{U}, \quad \forall j \in V, \\
x_{i j}^{\prime} & \leq y_{i}^{\prime}, \quad \forall i, j \in V, \\
\sum_{i \in V} w_{i} y_{i}^{\prime} & \leq 1 .
\end{aligned}
$$

Moreover, $y_{i}^{\prime}=1$ for all $i \in U$, and $y_{i}^{\prime}=0$ for all big vertices $i \in V \backslash U$. Thus, the two extra fractional vertices opened by BAsicFRKNAPCEnTERRound have weight at most $\epsilon$. By Proposition 4.2, we have $w(\mathcal{S}) \leq 1+2 \epsilon$. Moreover, conditioned on $U$, we have

$$
\operatorname{Pr}[j \in C \mid \mathcal{E}(U)] \geq \sum_{i \in B_{j}} x_{i j}^{\prime}=\sum_{i \in B_{j}} x_{i j} / q_{U}
$$

Thus, by definition of $\mathcal{P}_{\text {config } 1}$ and our construction of $\mathcal{S}$, we get

$$
\operatorname{Pr}[j \in C]=\sum_{U \in \mathcal{U}} \operatorname{Pr}[j \in C \mid \mathcal{E}(U)] \operatorname{Pr}[\mathcal{E}(U)] \geq \sum_{U \in \mathcal{U}} \sum_{i \in B_{j}} x_{i j} \geq p_{j}
$$

4.2.3 An Algorithm that Satisfies the Knapsack Constraint Exactly. Let $\epsilon>0$ a small parameter to be determined. Let $\mathcal{U}$ denote the collection of all possible vertex sets of size at most $\lceil 1 / \epsilon\rceil$ that have weight at most 1 ; note that $|\mathcal{U}| \leq n^{O(1 / \epsilon)}$. Suppose $R$ is the optimal radius to our instance. Given a set $U \in \mathcal{U}$, we say that vertex $j \in V$ is blue if $d(j, U) \leq 3 R$; otherwise, vertex $i$ is red. For any $i \in V$, let $\mathrm{RBall}(i, U, R)$ denote the set of red vertices within radius $3 R$ from $i$ :

$$
\operatorname{RBall}(i, U, R):=\{j \in V: d(i, j) \leq 3 R \wedge d(j, U)>3 R\} .
$$

Consider the configuration polytope $\mathcal{P}_{\text {confige }}$ containing points $(x, y, q)$ with the following constraints:

$$
\begin{cases}\sum_{U \in \mathcal{U}} q_{U}=1, & \\ \sum_{i \in B_{j}} x_{i j}^{U} \leq q_{U}, & \forall j \in V, U \in \mathcal{U} \\ \sum_{U \in \mathcal{U}} \sum_{i \in B_{j}} x_{i j}^{U} \geq p_{j}, & \forall j \in V \\ x_{i j}^{U} \leq y_{i}^{U}, & \forall i, j \in V, U \in \mathcal{U} \\ \sum_{i \in V} w_{i} y_{i}^{U} \leq q_{U}, & \forall U \in \mathcal{U} \\ \sum_{j \in V} \sum_{i \in B_{j}} x_{i j}^{U} \geq q_{U} t, & \\ y_{i}^{U}=1, & \forall U \in \mathcal{U}, i \in U \\ y_{i}^{U}=0, & \forall U \in \mathcal{U}, i \in V \backslash U,|\operatorname{RBall}(i, U, R)| \geq \epsilon n \\ x_{i j}^{U}, y_{i}^{U}, q_{U} \geq 0, & \forall i, j \in V, U \in \mathcal{U} .\end{cases}
$$

ACM Transactions on Algorithms, Vol. 15, No. 3, Article 36. Publication date: June 2019. 
We first claim that $\mathcal{P}_{\text {config2 }}$ is a valid relaxation polytope for the problem.

Proposition 4.4. The polytope $\mathcal{P}_{\text {config } 2}$ is non-empty.

Proof. Suppose $\mathcal{S}$ is a solution drawn from the optimal distribution $\mathcal{D}$. Consider the following randomized procedure to generate a random subset $U_{\mathcal{S}}$ of $\mathcal{S}$ :

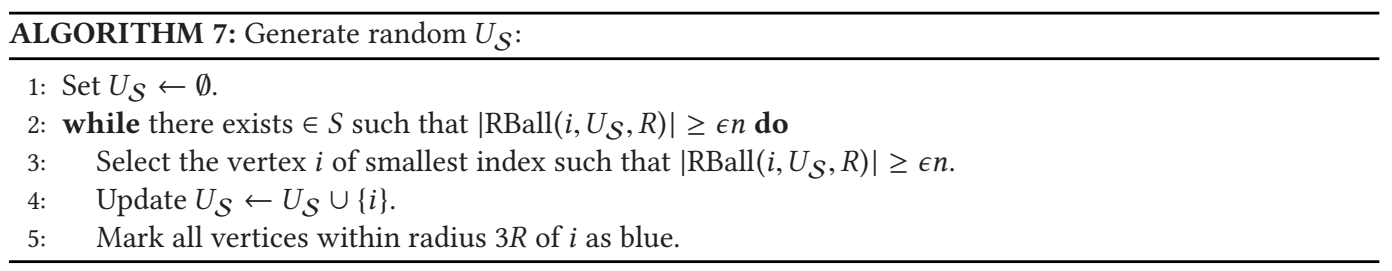

Note that for all $i \in \mathcal{S} \backslash U_{\mathcal{S}}$, we have $\left|\operatorname{RBall}\left(i, U_{\mathcal{S}}, R\right)\right|<\epsilon n$ by the condition of the while-loop. Moreover, we claim that $\left|U_{\mathcal{S}}\right| \leq\lceil 1 / \epsilon\rceil$, so that $U_{\mathcal{S}} \in \mathcal{U}$. For, suppose $\left|U_{\mathcal{S}}\right|>1 / \epsilon$; for each $i \in U_{\mathcal{S}}$, there are at least $\epsilon n$ red vertices turned into blue by $i$ in the procedure. This implies that there are more than $(1 / \epsilon) \times \epsilon n=n$ vertices, which is a contradiction.

Now for any $U \in \mathcal{U}$, we set $q_{U}:=\operatorname{Pr}\left[U_{\mathcal{S}}=U\right]$. Let $x_{i j}^{U}=\operatorname{Pr}\left[U_{S}=\mathcal{U} \wedge j\right.$ is connected to $\left.i\right]$, and finally let $y_{i}^{U}=\operatorname{Pr}\left[U_{\mathcal{S}}=U \wedge i \in \mathcal{S}\right]$. Then it is clear that $\sum_{U \in \mathcal{U}} q_{U}=1$. Using similar arguments as in the proof of Proposition 4.3, we have the following inequalities:

$$
\begin{aligned}
& \sum_{i \in B_{j}} x_{i j}^{U} \leq q_{U}, \quad \forall j \in V, U \in \mathcal{U} \\
& \sum_{U \in \mathcal{U}} \sum_{i \in B_{j}} x_{i j}^{U} \geq p_{j}, \quad \forall j \in V \\
& \sum_{i \in V} w_{i} y_{i}^{U} \leq q_{U}, \quad \forall U \in \mathcal{U} \\
& \sum_{j \in V} \sum_{i \in B_{j}} x_{i j}^{U} \geq q_{U} t .
\end{aligned}
$$

As mentioned before, if $\left|\operatorname{RBall}\left(i, U_{\mathcal{S}}, R\right)\right| \geq \epsilon n$, then $i \notin \mathcal{S}$. Therefore,

$$
y_{i}^{U}=\operatorname{Pr}\left[U_{\mathcal{S}}=U \wedge i \in \mathcal{S}\right]=0, \quad \forall U \in \mathcal{U}, i \in V \backslash U,|\operatorname{RBall}(i, U, R)| \geq \epsilon n .
$$

The other constraints can be verified easily. We conclude that $(x, y, q) \in \mathcal{P}_{\text {config2 }}$.

Next, let us pick any $(x, y, q) \in \mathcal{P}_{\text {config2 }}$ and use the following algorithm to round it.

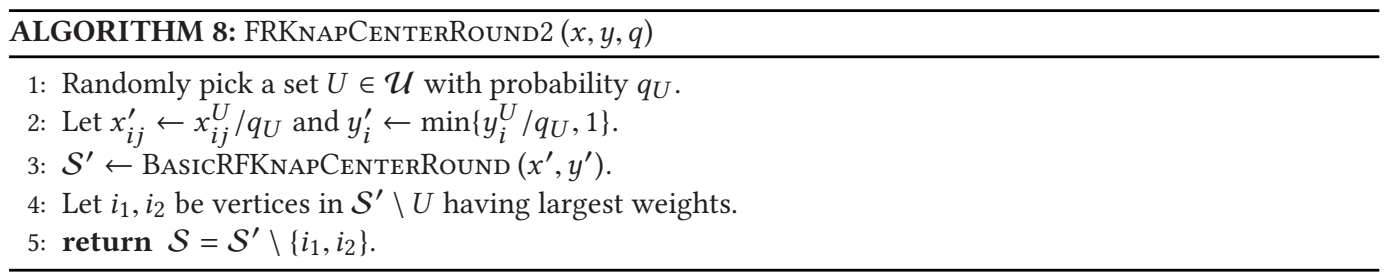


Analysis. Let us fix any $\gamma>0$ and set $\epsilon:=\frac{\gamma^{2}}{2}$. Also, let $\mathcal{E}(U)$ denote the event that $U \in \mathcal{U}$ is picked in the algorithm. Again, observe that $\left(x^{\prime}, y^{\prime}\right)$ satisfies the following inequalities:

$$
\begin{aligned}
\sum_{j \in V} \sum_{i \in B_{j}} x_{i j}^{\prime} & \geq t, \\
\sum_{i \in B_{j}} x_{i j}^{\prime} & \leq 1, \quad \forall j \in V, \\
\sum_{i \in B_{j}} x_{i j}^{\prime} & =\sum_{i \in B_{j}} x_{i j} / q_{U}, \quad \forall j \in V, \\
x_{i j}^{\prime} & \leq y_{i}^{\prime}, \quad \forall i, j \in V, \\
\sum_{i \in V} w_{i} y_{i}^{\prime} & \leq 1 .
\end{aligned}
$$

Recall that the algorithm BASICFRKNAPCENTERROUnD will return a solution $\mathcal{S}^{\prime}$ consisting of a set $\mathcal{S}^{\prime \prime}$ with $w\left(\mathcal{S}^{\prime \prime}\right) \leq 1$ plus (at most) two extra "fractional" centers $i^{*}$ and $i^{* *}$. Since $y_{i^{* *}}^{\prime}, y_{i^{*}}^{\prime}$ are fractional, we have that $i^{*}, i^{* *} \notin U$. Thus, by removing the two centers having highest weights in $\mathcal{S}^{\prime} \backslash U$, we ensure that $w(\mathcal{S}) \leq 1$ with probability one.

Now we shall prove the coverage guarantee. By Proposition 4.2, $\mathcal{S}^{\prime}$ covers at least $t$ vertices within radius $3 R$. If a vertex is blue, it can always be connected to some center in $U$ and so it is not affected by the removal of $i_{1}, i_{2}$. Because each of $i_{1}$ and $i_{2}$ can cover at most $\epsilon n$ other red vertices, we have

$$
|C| \geq t-2 \epsilon n=t-\gamma^{2} n .
$$

For any $j \in V$, let $X_{j}$ be the indicator random variable for the event that $d\left(j, \mathcal{S}^{\prime}\right) \leq 3 R$ but $d(j, \mathcal{S} \backslash$ $\left.\left\{i_{1}, i_{2}\right\}\right)>3 R$. We say that $j$ is a bad vertex iff $\mathrm{E}\left[X_{j}\right] \geq \gamma$, otherwise, vertex $j$ is good. Note that $\sum_{j \in V} X_{j} \leq 2 \epsilon n$ with probability one. Thus, there can be at most $2 \epsilon n / \gamma$ bad vertices. Letting $T$ denote the set of good vertices, we have

$$
|T| \geq n-2 \epsilon n / \gamma=(1-\gamma) n .
$$

By Proposition 4.2, $\operatorname{Pr}\left[j\right.$ is covered by $\left.\mathcal{S}^{\prime}\right] \geq p_{j}$. For any $j \in T$, we have

$$
\begin{aligned}
\operatorname{Pr}[j \in C] & =\operatorname{Pr}\left[j \text { is covered by } \mathcal{S}^{\prime} \wedge X_{j}=0\right] \\
& =\operatorname{Pr}\left[j \text { is covered by } \mathcal{S}^{\prime}\right]-\operatorname{Pr}\left[j \text { is covered by } \mathcal{S}^{\prime} \wedge X_{j}=1\right] \\
& \geq \operatorname{Pr}\left[j \text { is covered by } \mathcal{S}^{\prime}\right]-\operatorname{Pr}\left[X_{j}=1\right] \geq p_{j}-\gamma .
\end{aligned}
$$

This concludes the first part of Theorem 1.4 for the FRKnapCenter problem.

\section{THE MATROID CENTER PROBLEMS WITH OUTLIERS}

In this section, we will first give a tight 3-approximation algorithm for the RMatCenter problem, improving upon the 7-approximation algorithm by Chen et al. [5]. Then we study the FRMatCenter problem and prove the second part of Theorem 1.4.

\subsection{Matroid Polytopes}

We first review a few basic facts about matroid polytopes. For any vector $z$ and set $S$, we let $z(S)$ denote the sum $\sum_{i \in S} z_{i}$. Let $\mathcal{M}$ be any matroid on the ground set $\Omega$ and $r_{\mathcal{M}}$ be its rank function. The matroid polytope of $\mathcal{M}$ is defined by

$$
\mathcal{P}_{\mathcal{M}}:=\left\{x \in \mathbb{R}^{\Omega}: x(S) \leq r_{\mathcal{M}}(S) \forall S \subseteq \Omega ; \quad x_{i} \geq 0 \forall i \in \Omega\right\}
$$


and the matroid base polytope of $\mathcal{M}$ is defined by

$$
Q_{\mathcal{M}}:=\left\{x \in \mathbb{R}^{\Omega}: x(S) \leq r_{\mathcal{M}}(S) \forall S \subseteq \Omega ; \quad x(\Omega)=r_{\mathcal{M}}(\Omega) \quad x_{i} ; \geq 0 \quad \forall i \in \Omega\right\} .
$$

Definition 5.1. Suppose $A x \leq b$ is a valid inequality of a polytope $\mathcal{P}$. A face $D$ of $\mathcal{P}$ (corresponding to this valid inequality) is the set $D:=\{x \in \mathcal{P}: A x=b\}$.

The following theorem gives a characterization for any face of $\mathcal{P}_{\mathcal{M}}$ (see Section 5.2.2 in [12]).

TheOREM 5.2. Let $D$ be any face of $\mathcal{P}_{\mathcal{M}}$. Then it can be characterized by

$$
D=\left\{x \in \mathbb{R}^{\Omega}: x(S)=r_{\mathcal{M}}(S) \forall S \in \mathcal{L} ; \quad x_{i}=0 \forall i \in J ; \quad x \in \mathcal{P}_{\mathcal{M}}\right\},
$$

where $J \subseteq \Omega$ and $\mathcal{L}$ is a chain family of sets: $L_{1} \subset L_{2} \subset \ldots \subset L_{m}$. Moreover, it is sufficient to choose $\mathcal{L}$ as any maximal chain $L_{1} \subset L_{2} \subset \ldots \subset L_{m}$ such that $x\left(L_{i}\right)=r_{\mathcal{M}}\left(L_{i}\right)$ for all $i=1,2, \ldots, m$.

Proposition 5.3. Let $x \in \mathcal{P}_{\mathcal{M}}$ be any point and I be the set of all tight constraints of $\mathcal{P}_{\mathcal{M}}$ on $x$. Suppose $D$ is the face with respect to $I$. Then one can compute a chain family $\mathcal{L}$ for $D$ as in Theorem 5.2 in polynomial time.

Proof. Recall that $r_{\mathcal{M}}$ is a submodular function. Thus the function $r_{\mathcal{M}}^{\prime}(S)=r_{\mathcal{M}}(S)-x(S)$ for $S \subseteq \Omega$ is also submodular. It is well-known that submodular minimization can be done in polynomial time (see, e.g., Theorem 45.1 in [14]). We solve the following optimization problem: $\min \left\{r_{\mathcal{M}}^{\prime}(S): S \subseteq \Omega\right\}$. If there are multiple solutions, we let $S_{0}$ be any solution of minimal size. (This can be done easily, say, by trying to drop each item from the current solution and resolving the program.) We add $S_{0}$ to our chain. Then we find some minimal superset $S_{1}$ of $S_{0}$ such that $r_{\mathcal{M}}^{\prime}\left(S_{1}\right)=0$, add $S_{1}$ to our chain, and repeat the process.

Corollary 5.4. Let $D$ be any face of $\mathcal{P}_{\mathcal{M}}$. Then it can be characterized by

$$
D=\left\{x \in \mathbb{R}^{\Omega}: x(S)=b_{S} \quad \forall S \in O ; \quad x_{i}=0 \forall i \in J ; \quad x \in \mathcal{P}_{\mathcal{M}}\right\},
$$

where $J \subseteq \Omega$ and $O$ is a family of pairwise disjoint sets: $O_{1}, O_{2}, \ldots, O_{m}$, and $b_{O_{1}}, \ldots, b_{O_{m}}$ are some integer constants.

Proof. By Theorem 5.2, we have

$$
D=\left\{x \in \mathbb{R}^{\Omega}: x(S)=r_{\mathcal{M}}(S) \forall S \in \mathcal{L} ; \quad x_{i}=0 \forall i \in J ; \quad x \in \mathcal{P}_{\mathcal{M}}\right\},
$$

where $J \subseteq \Omega$ and $\mathcal{L}$ is the chain: $L_{1} \subset L_{2} \subset \ldots \subset L_{m}$. Now let us define $O_{1}:=L_{1}, O_{2}:=L_{2} \backslash$ $L_{1}, O_{3}:=L_{3} \backslash L_{2}, \ldots, O_{m}:=L_{m} \backslash L_{m-1}$, and $b_{O_{1}}:=r_{\mathcal{M}}\left(L_{1}\right), b_{O_{2}}:=r_{\mathcal{M}}\left(L_{2}\right)-r_{\mathcal{M}}\left(L_{1}\right), \ldots, b_{O_{m}}:=$ $r_{\mathcal{M}}\left(L_{m}\right)-r_{\mathcal{M}}\left(L_{m-1}\right)$. It is not difficult to verify that

$$
D=\left\{x \in \mathbb{R}^{\Omega}: x(S)=b_{S} \quad \forall S \in O ; \quad x_{i}=0 \forall i \in J ; \quad x \in \mathcal{P}_{\mathcal{M}}\right\} .
$$

Theorem 5.5 (Theorem 2 In [6]). Let $D$ be any face of the matroid base polytope $Q_{\mathcal{M}}$. Then $D$ itself is a matroid base polytope of some matroid $\mathcal{M}^{\prime}$ on the same ground set $\Omega$.

\subsection{The Robust Matroid Center Problem}

Suppose $\mathcal{I}=(V, d, \mathcal{M}, t)$ is an instance of the RMatCenter problem with optimal radius $R$. Let $r_{\mathcal{M}}$ denote the rank function of $\mathcal{M}$. Consider the polytope $\mathcal{P}_{\text {RMatCenter containing points }}(x, y)$ satisfying constraints (1)-(4), and the matroid rank constraints:

$$
y(U) \leq r_{\mathcal{M}}(U), \quad \forall U \subseteq V .
$$

While $\mathcal{P}_{\mathrm{R} M a t C e n t e r}$ has exponential many constraints in the definition, we can still verify whether $\mathcal{P}_{\mathrm{R} M a t C e n t e r} \neq \emptyset$ (when performing the binary search on $R$ ) and compute a solution 
$(x, y) \in \mathcal{P}_{\mathrm{RMatCenter}}$ if it is non-empty in polynomial time. This can be done because there is an efficient separation oracle for $\mathcal{P}_{\mathrm{RMatC}}$ nter. Specifically, the most violated matroid rank constraint can be found via submodular minimization. The next step is to round $(x, y)$ into an integral solution. Our 3-approximation algorithm is as follows.

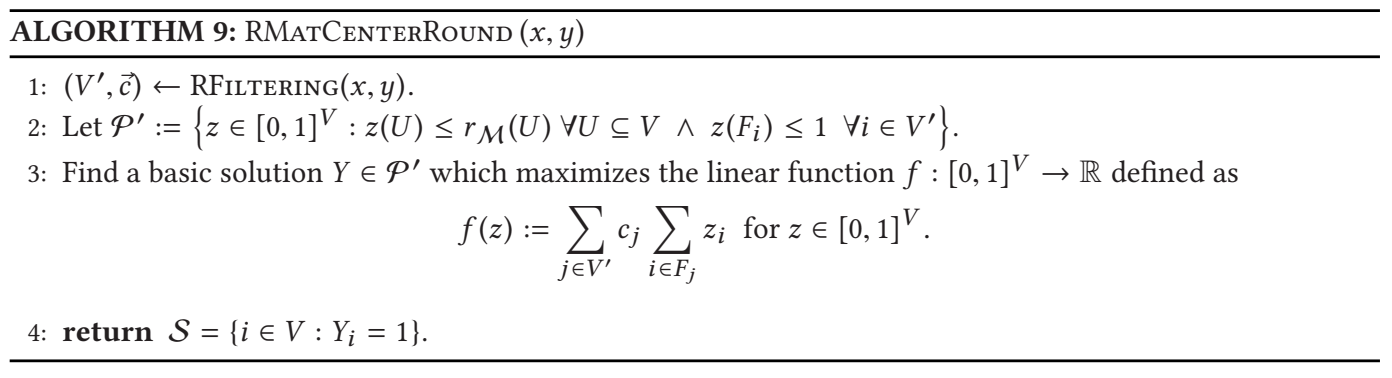

Analysis. Again, by construction, the clusters $F_{i}$ are pairwise disjoint for $i \in V^{\prime}$. Note that $\mathcal{P}^{\prime}$ is the matroid intersection polytope between $\mathcal{M}$ and another partition matroid polytope saying that at most one item per set $F_{i}$ for $i \in V^{\prime}$ can be chosen. Moreover, we shall prove in Proposition 5.6 that $\mathcal{P}^{\prime} \neq \emptyset$. Thus, $\mathcal{P}^{\prime}$ has integral extreme points and optimizing over $\mathcal{P}^{\prime}$ can be done in polynomial time. Note that the solution $\mathcal{S}$ is feasible as it satisfies the matroid constraint. The correctness of RMATCENTERRound follows immediately by the following two propositions.

Proposition 5.6. We have $\mathcal{P}^{\prime} \neq \emptyset$ and $f(Y) \geq t$.

Proof. For each $j \in V^{\prime}$ and $i \in F_{j}$, define $y_{i}^{\prime}:=x_{i j}$ (this is well-defined as all clusters $F_{j}$ for $j \in V^{\prime}$ are pairwise disjoint). Also, set $y_{i}^{\prime}:=0$ for other vertices $i$ not belonging to any marked cluster. By the definitions of $\mathcal{P}_{\mathrm{R} M a t C e n t e r}$ and $\mathcal{P}^{\prime}$, one can verify that $y^{\prime} \in \mathcal{P}^{\prime}$, implying that $\mathcal{P}^{\prime} \neq \emptyset$.

Next, by greedy choice and constraint (1), we have

$$
f\left(y^{\prime}\right)=\sum_{j \in V^{\prime}} c_{j} y^{\prime}\left(F_{j}\right)=\sum_{j \in V^{\prime}} c_{j} s_{j} \geq \sum_{j \in V} s_{j} \geq t .
$$

By the choice of $Y$, we have $f(Y) \geq f\left(y^{\prime}\right) \geq t$.

Proposition 5.7. There are at least $f(Y)$ vertices in $V$ that are at distance at most $3 R$ from some open center in $\mathcal{S}$.

Proof. Recall that $\mathcal{S}$ is the set of vertices $i \in V$ such that $Y_{i}=1$. Moreover, since $Y\left(F_{j}\right) \leq 1$, there can be at most one open center in $F_{j}$ (i.e., $\left|\mathcal{S} \cap F_{j}\right| \leq 1$ ) for each $j \in V^{\prime}$. For any $j \in V^{\prime}$,

-if $Y\left(F_{j}\right)=0$, then there is no open center in $F_{j}$ and its contribution in $f(Y)$ is zero;

-if $Y\left(F_{j}\right)=1$, then we open some center $i \in F_{j}$ and the contribution of $j$ to $f(Y)$ is equal to $c_{j}$. (Recall that $c_{j}$ is the number of clusters $F_{k}$ with $F_{j} \cap F_{k} \neq \emptyset$.) By triangle inequality, $d(k, i) \leq d(k, j)+d(j, i) \leq 2 R+R=3 R$.

This analysis proves the second part of Theorem 1.1.

\subsection{The Fair Robust Matroid Center Problem}

In this section, we consider the FRMatCenter problem. It is not difficult to modify and randomize algorithm RMCENTERROUND so that it would return a random solution satisfying both the fairness guarantee and matroid constraint, and preserving the coverage constraint in expectation. This can be done by randomly picking $Y$ inside $\mathcal{P}^{\prime}$. However, if we want to obtain some concrete guarantee 


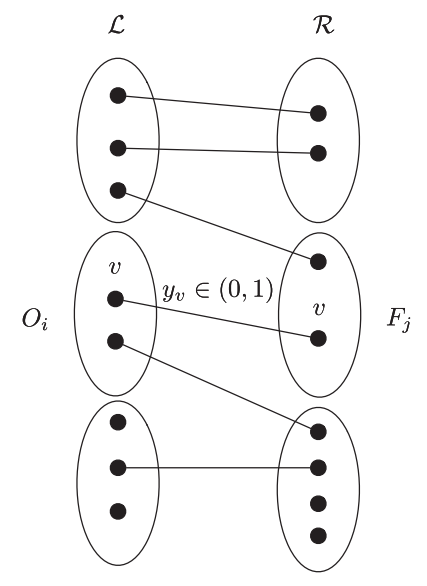

Fig. 1. Construction of the multi-bipartite graph $H=\left(\mathcal{L}, \mathcal{R}, E_{H}\right)$ in the main algorithm.

on the coverage constraint, we may have to (slightly) violate either the matroid constraint or the fairness guarantee. We leave it as an open question whether there exists a true approximation algorithm for this problem.

We will start with a pseudo-approximation algorithm which always returns a basis of $\mathcal{M}$ plus at most one extra center. Our algorithm is quite involved. We first carefully round a fractional solution inside a matroid intersection polytope into a (random) point with a special property: the unrounded variables form a single path connecting some clusters and tight matroid rank constraints. Next, rounding this point will ensure that all but one cluster has an open center. Then opening one extra center is sufficient to cover at least $t$ clients.

Finally, using a similar preprocessing step similar to the one in Section 4.2.3, we correct the solution by removing the extra center without affecting the fairness and coverage guarantees by too much. This algorithm concludes Theorem 1.4.

5.3.1 A Pseudo-Approximation Algorithm. Suppose $\mathcal{I}=(V, d, \mathcal{M}, t, \vec{p})$ is an instance of the robust matroid center problem with the optimal radius $R$. Let $r_{\mathcal{M}}$ denote the rank function of $\mathcal{M}$ and $\mathcal{P}_{\mathcal{M}}$ be the matroid base polytope of $\mathcal{M}$. Consider the polytope $\mathcal{P}_{\text {FRMatCenter containing points }}$ $(x, y)$ satisfying constraints (1)-(4), the fairness constraint (5), and the matroid constraints (8). We note that $\mathcal{P}_{\text {FRMatCenter }}$ is a valid relaxation.

The main algorithm is summarized in Algorithm 10, which can round any vertex point $(x, y) \in$ $\mathcal{P}_{\text {FRMatCenter }}$. Basically, we will round $y$ iteratively. In each round, we construct a (multi)-bipartite graph where vertices on the left side are the disjoint sets $O_{1}, O_{2}, \ldots$ in Corollary 5.4. Vertices on the right side are corresponding to the disjoint sets $F_{1}, F_{2}, \ldots$ returned by RFILTERING. Now each edge of the bipartite graph, connecting $O_{i}$ and $F_{j}$, represents some unrounded variable $y_{v} \in(0,1)$ where $v \in O_{i}$ and $v \in F_{j}$ (see Figure 1).

Then we carefully pick a cycle (path) on this graph and round variables on the edges of this cycle (path). This is done by subroutines RoundCycle, RoundSinglePath, and RoundTwoPaths. See Figures 2, 3, and 4. Basically, these procedures will first choose a direction $\vec{r}$ which alternatively increases and decreases the variables on the cycle (path) so that (i) all tight matroid constraints are preserved and (ii) the number of (fractionally) covered clients is also preserved. Now we randomly move $y$ along $\vec{r}$ or $-\vec{r}$ to ensure that all the marginal probabilities are preserved.

Finally, all the remaining, fractional variables will form one path on the bipartite graph. We round these variables by the procedure RoundFinALPATH which exploits the integrality of any 


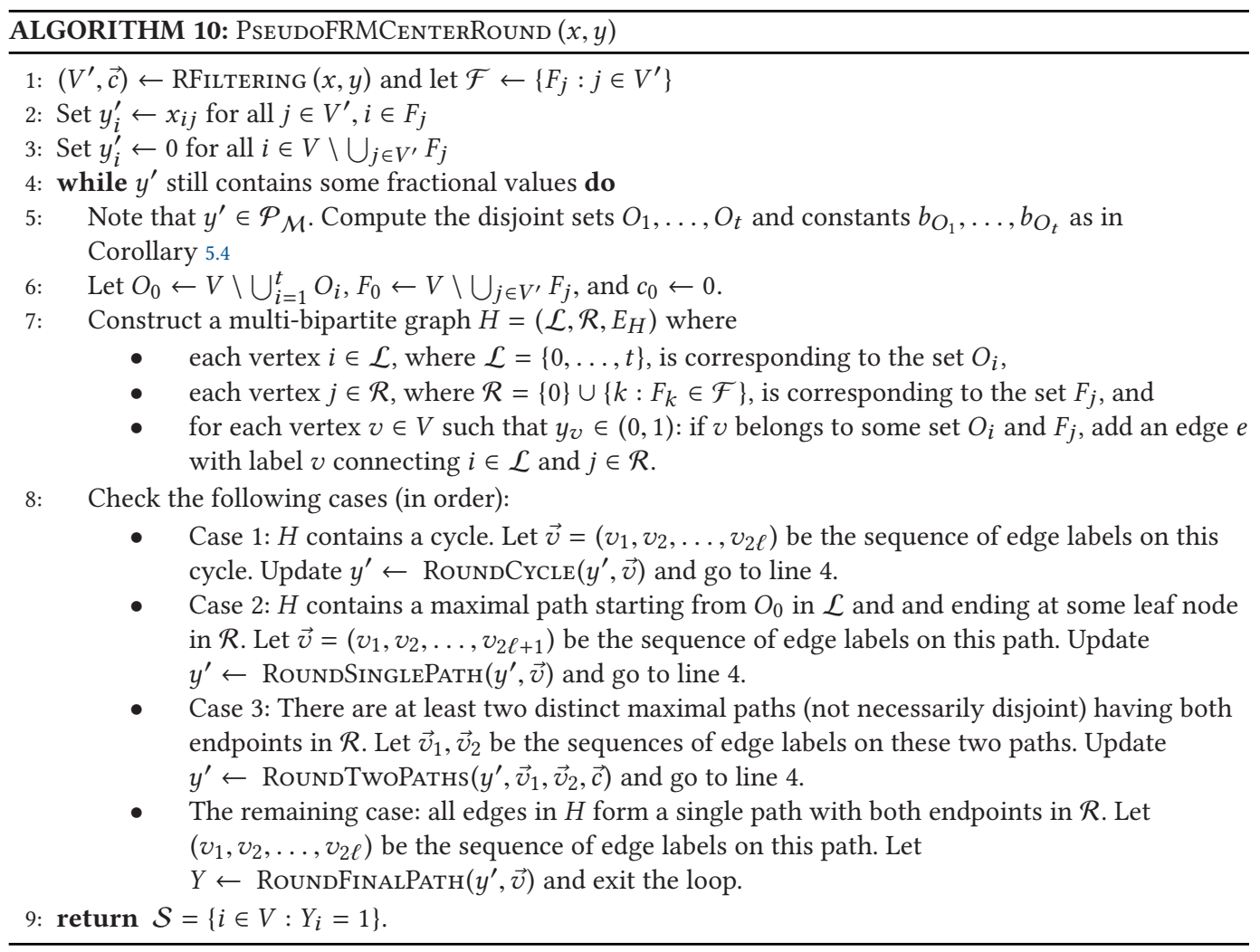

face of a matroid intersection polytope. Then, to cover at least $t$ clients, we may need to open one extra facility.

Proposition 5.8. The polytyope $\mathcal{P}_{\text {FRMatCenter }}$ is non-empty.

Proof. The proof is similar to Proposition 3.1, and is omitted.

We now explain how to implement the rounding steps in this algorithm. These are all based on the subroutine RoundSINGLEPoint, which moves the vector $y$ as far as possible along the direction of vector $\vec{r}$ until it hits a new face of the polytope. We define it formally as Algorithm 11; note that it can be implemented efficiently by solving an appropriate LP.

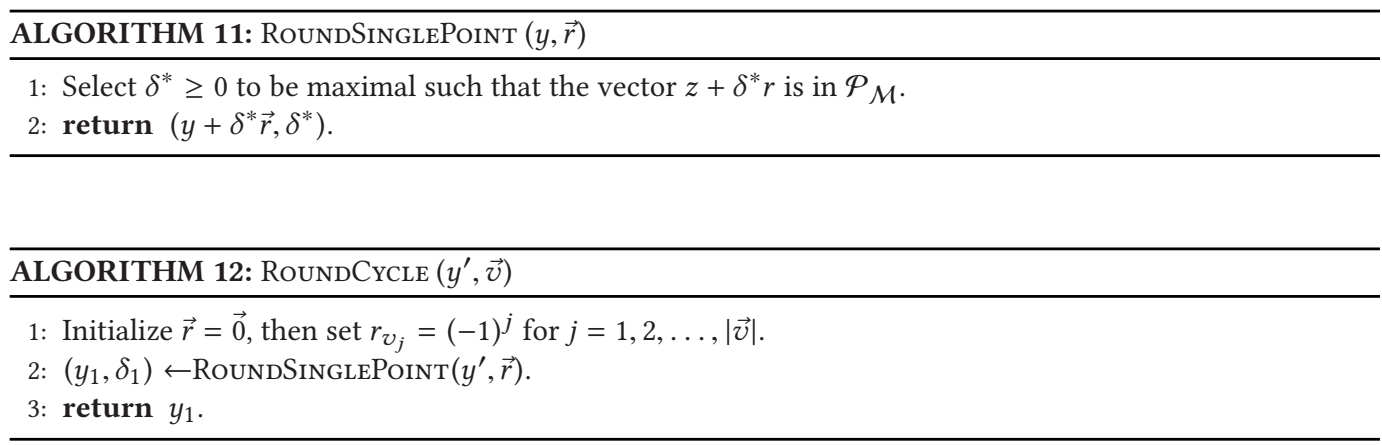



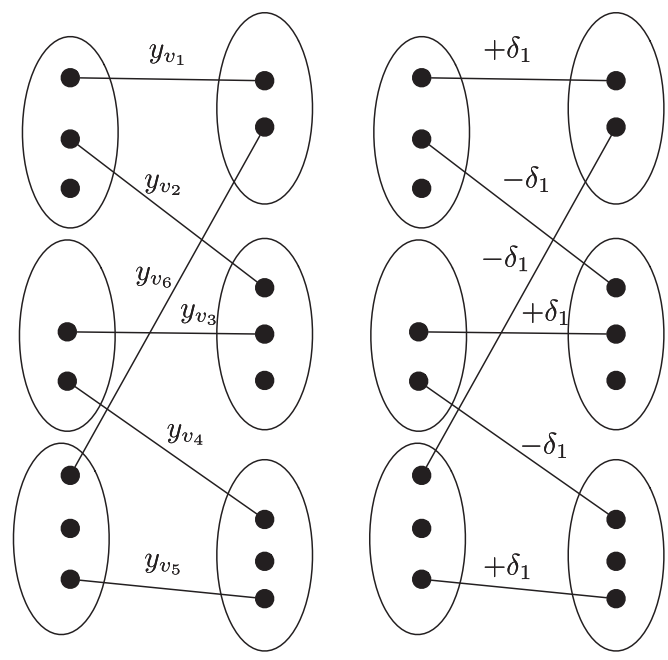

Fig. 2. The left part shows a cycle. The right part shows how the variables on the cycle are being changed by ROUNDCYCLE.
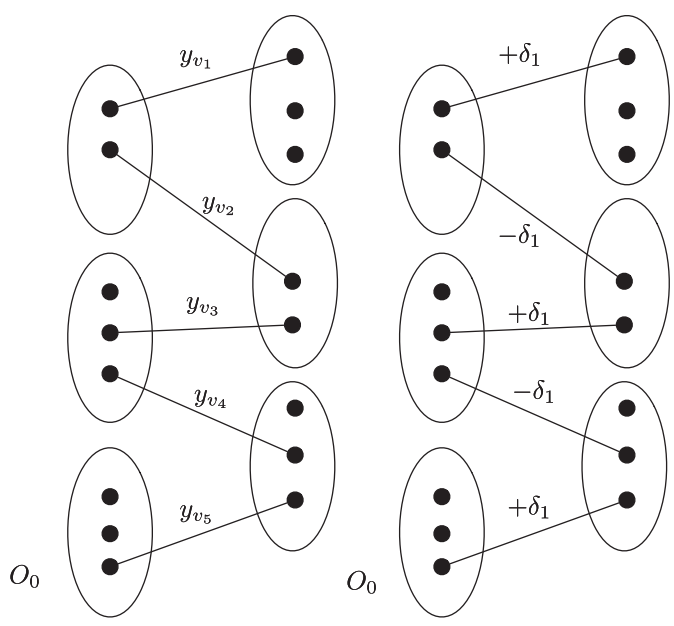

Fig. 3. The left part shows a single path. The right part shows how the variables on the path are being changed by RoundSINGLEPATH.

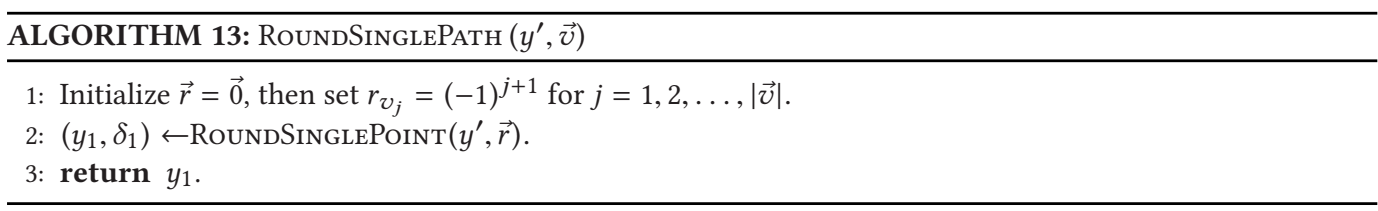

\subsubsection{Analysis of PSEUdoFRMCENTERROUND.}

Proposition 5.9. In all but the last iteration, the while-loop (lines 4-8) of PSEUDOFRMCENTERRound preserves the following invariant: if $y^{\prime}$ lies in the face $D$ of $\mathcal{P}_{\mathcal{M}}$ (w.r.t. all tight matroid rank constraints) at the beginning of the iteration, then $y^{\prime} \in D$ at the end of this iteration. 

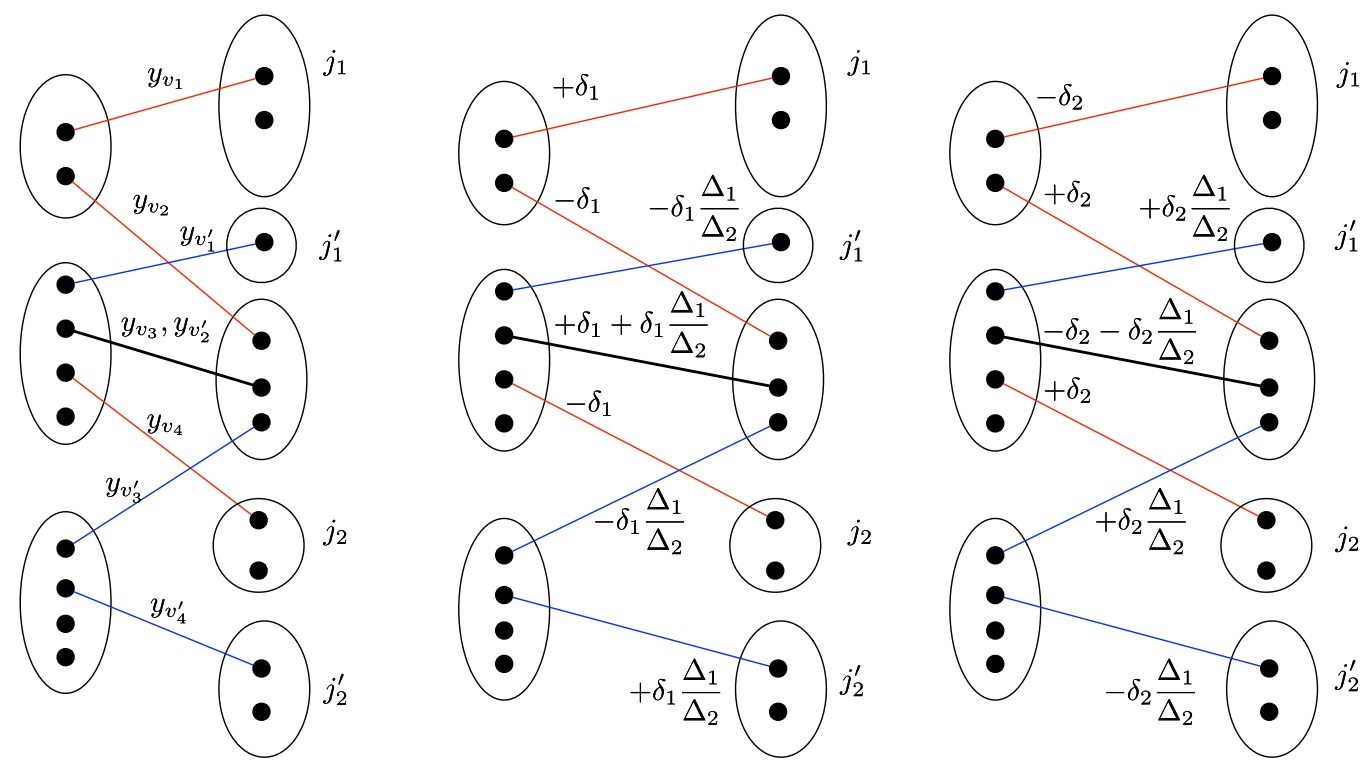

Fig. 4. The left part shows an example of two distinct maximal paths chosen in Case 3. The black edge is common in both paths. The middle and right parts are two possibilities of rounding $y$. With probability $\delta_{1} /\left(\delta_{1}+\delta_{2}\right)$, the strategy in the right part is adopted. Otherwise, the strategy in the middle part is chosen.

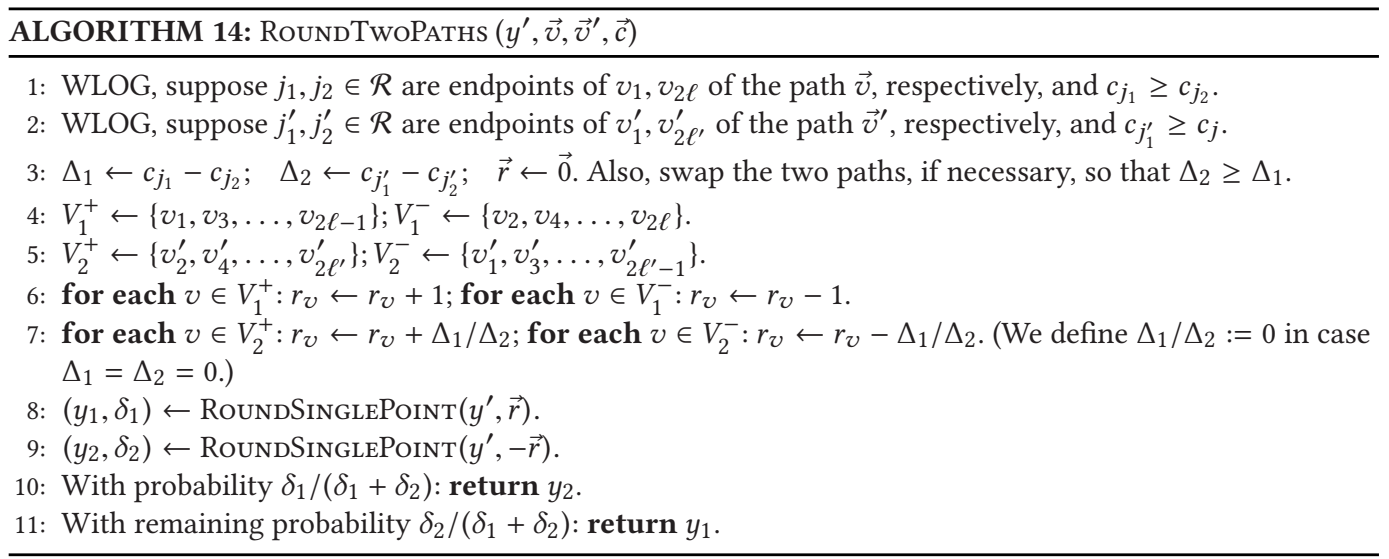

Proof. Observe that $y^{\prime} \in \mathcal{P}_{\mathcal{M}}$ at the beginning of the first iteration due to the definition of $y^{\prime}$. Fix any iteration. Let $y^{\prime \prime}$ be the updated $y^{\prime}$ at the end of the iteration. By Corollary 5.4, it suffices to show that

$$
y^{\prime \prime} \in\left\{x \in \mathbb{R}^{n}: x(S)=b_{S} \quad \forall S \in O ; \quad x_{i}=0 \quad \forall i \in J ; \quad x \in \mathcal{P}_{\mathcal{M}}\right\}
$$

where $J \subseteq V$ is the set of all vertices $i$ with $y_{i}^{\prime}=0$. Note that $y^{\prime \prime}$ is the output of one of the three subroutines RoundCycle, RoundSinglePath, and RoundTwoPaths. Since we only round floating variables strictly greater than zero, we have $y_{i}^{\prime \prime}=0$ for all $i \in J$. Also, the procedure RoundSinGLEPoint guarantees that $y^{\prime \prime} \in \mathcal{P}_{\mathcal{M}}$. 


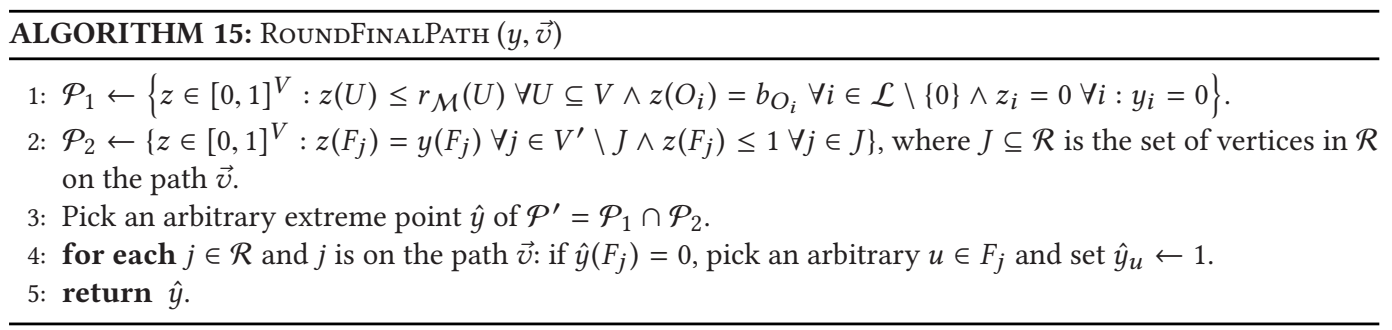

-When calling the procedure RoundCyCLE, observe that each vertex $j \in \mathcal{L}$ on the cycle is adjacent to exactly two edges. By construction, we always increase the variable on one edge and decrease the variable on the other edge at the same rate. See Figure 2. Therefore, $y^{\prime \prime}\left(O_{j}\right)=y^{\prime}\left(O_{j}\right)=b_{O_{j}}$ for all $j \in O$.

- When calling the procedure RoundSingLePATH, recall that our path is maximal and has one endpoint being $O_{0}$ and the other in $\mathcal{R}$. While $y^{\prime \prime}\left(O_{0}\right)$ may not be equal to $y^{\prime}\left(O_{0}\right)$, we do not violate any constraint as there is no restriction on the mass of $O_{0}$. By the same argument as before, we have $y^{\prime \prime}\left(O_{j}\right)=y^{\prime}\left(O_{j}\right)=b_{O_{j}}$ for all $j \in O$.

- In the procedure RoundTwoPATHs, we round the variables on two paths which have both endpoints in $\mathcal{R}$. Thus, any vertex $j$ should be adjacent to either 2 or 4 edges. Again, by construction, the net change in $y^{\prime}\left(O_{j}\right)$ is equal to zero. See Figure 4.

Finally, the claim follows by induction.

Proposition 5.10. PSeudoFrMCENTERRound terminates in polynomial time.

Proof. Note that, in each iteration, each floating variable $y_{v}^{\prime} \in(0,1)$ corresponds to exactly one edge in the bipartite graph. This is because, by construction, the sets $O_{0}, \ldots, O_{t}$ form a partition of $V$ and the sets in $\mathcal{F}$ and $F_{0}$ also form a partition of $V$. Thus, as long as there are fractional values in $y^{\prime}$, our graph will have some cycle or path.

Now we will show that the while-loop (lines 4-8) terminates after $O(|V|)$ iterations. First, observe that we actually cover all possible cases at line 8 . In particular, if there is no maximal path starting from $O_{0}$ and ending at some leaf node in $\mathcal{R}$, then all the remaining maximal paths should have both endpoints in $\mathcal{R}$. Otherwise, suppose there exists a maximal path starting at $O_{j}$ with $j \neq 0$. We have the tight constraint $y^{\prime}\left(O_{j}\right)=b_{O_{j}} \in \mathbb{Z}^{+}$. Then the degree of the vertex $j$ must be at least 2 as there must be at least two fractional variables in this set. This contradicts the fact that our path is maximal.

Next, for any set $S$, let $\chi(S)$ denote the characteristic vector of $S$. That is, $\chi(v)=1$ for $v \in S$ and $\chi(v)=0$ otherwise. Let us fix any iteration and let $\mathcal{T}=\left\{\chi(S): S \subseteq V \wedge y^{\prime}(S)=r_{\mathcal{M}}(S)\right\}$ be the set of all tight constraints. In this iteration, we will move $y^{\prime}$ along some direction $\vec{r}$ as far as possible (by procedure RoundSinglePoint). It means that the new point $y^{\prime \prime}=y^{\prime}+\delta^{*} \vec{r}$ will either have at least one more rounded variable or hit a new tight constraint $y^{\prime \prime}\left(S_{0}\right)=r_{\mathcal{M}}\left(S_{0}\right)$ (while $\left.y^{\prime}\left(S_{0}\right)<r_{\mathcal{M}}\left(S_{0}\right)\right)$ for some $S_{0} \subseteq V$. Indeed, $\chi\left(S_{0}\right)$ is linearly independent of all vectors in $\mathcal{T}$.

Proposition 5.9 says that all the tight constraints are preserved in the rounding process. Therefore, in the next iteration, we either have at least one more rounded variable or the rank of $\mathcal{T}$ is increased by at least 1 . This implies the algorithm terminates after at most $|V|$ iterations.

Proposition 5.11. In all iterations, the while-loop (lines 4-8) of PSEUDOFRMCENTERROUND satisfies the invariant that $y^{\prime}\left(F_{j}\right) \leq 1$ for all $F_{j} \in \mathcal{F}$.

Proof. By constraints 2 and 3, this property is true at the beginning of the first iteration. By a very similar argument as in the proof of Proposition 5.9, this is also true during all but the last 
iteration. (Note that if $j$ is an endpoint of a path, then $j$ must be adjacent to exactly one fractional value $y_{v}^{\prime}$, which could be rounded to one, while other variables $\left\{y_{v^{\prime}}^{\prime}: v^{\prime} \in F_{j}, v^{\prime} \neq v\right\}$ are already rounded to zero as our path is maximal.) Finally, it is not hard to check that procedure RoundFINALPATH also does not violate this invariant.

Proposition 5.12. PSEUdoFRMCENTERRound returns a solution $\mathcal{S}$ which is some independent set of $\mathcal{M}$ plus (at most) one extra vertex in $V$.

Proof. Let us focus on the procedure RoundFinalPath. Recall that the polytope $\mathcal{P}^{\prime}$ in RoundFinalPath is the intersection of the following two polytopes:

$$
\mathcal{P}_{1}=\left\{z \in[0,1]^{V}: z(U) \leq r_{\mathcal{M}}(U) \forall U \subseteq V \wedge z\left(O_{i}\right)=b_{O_{i}} \forall i \in \mathcal{L} \backslash\{0\} \wedge z_{i}=0 \forall i: y_{i}=0\right\},
$$

and

$$
\mathcal{P}_{2}=\left\{z \in[0,1]^{V}: z\left(F_{j}\right)=y\left(F_{j}\right) \forall j \in V^{\prime} \backslash J \wedge z\left(F_{j}\right) \leq 1 \forall j \in J\right\},
$$

where $J \subseteq \mathcal{R}$ is the set of vertices in $\mathcal{R}$ on the path $\vec{v}$.

First, we claim that $\mathcal{P}_{1}$ is a matroid base polytope. Let $\ell:=\sum_{i \in \mathcal{L} \backslash\{0\}} b_{O_{i}}$. Note that $\ell$ is an integer, which is less than or equal to the rank of $\mathcal{M}$. Let $\mathcal{T}$ be the family of independent sets of $\mathcal{M}$ having size at most $\ell$. Then we have that $\mathcal{M}^{\prime}=(\mathcal{T}, V)$ is a matroid with the matroid base polytope:

$$
\begin{aligned}
Q_{\mathcal{M}^{\prime}} & =\left\{z \in[0,1]^{V}: z(U) \leq \min \left\{r_{\mathcal{M}}(U), \ell\right\} \forall U \subseteq V \wedge z(V)=\ell\right\} \\
& =\left\{z \in[0,1]^{V}: z(U) \leq r_{\mathcal{M}}(U) \forall U \subseteq V \wedge z(V)=\ell\right\} .
\end{aligned}
$$

Now it is clear that $\mathcal{P}_{1}$ is a face of $Q_{\mathcal{M}^{\prime}}$. By Theorem 5.5, $\mathcal{P}_{1}$ itself is also a matroid base polytope.

By Propositions 5.9 and 5.11, we have $y \in \mathcal{P}_{1}$ and $y \in \mathcal{P}_{2}$. Thus, $y \in \mathcal{P}$, which implies that $\mathcal{P} \neq \emptyset$. Moreover, $\mathcal{P}_{2}$ is a partition matroid polytope. (Observe that $z\left(F_{j}\right)=y\left(F_{j}\right) \in\{0,1\} \forall j \in V^{\prime} \backslash J$ since all fractional variables are on the path $\vec{v}$.) Therefore, $\mathcal{P}=\mathcal{P}_{1} \cap \mathcal{P}_{2}$ has integral extreme points and the point $\hat{y}$ chosen in line 3 is integral.

Finally, recall that $\vec{v}=\left(v_{1}, v_{2}, \ldots, v_{2 \ell}\right)$ is a simple path with both endpoints in $\mathcal{R}$. Note that, by construction, $O_{0}$ is not on $\vec{v}$. The constraints of $\mathcal{P}_{1}$ and integrality of $b_{O_{i}}$ 's ensure that $\hat{y}_{v_{1}}+$ $\hat{y}_{v_{2}}=1, \hat{y}_{v_{3}}+\hat{y}_{v_{4}}=1, \ldots, \hat{y}_{v_{2 \ell-1}}+\hat{y}_{v_{2 \ell}}=1$. In other words, every vertex $i \in \mathcal{L}$ on the path will be "matched" with exactly one vertex in $\mathcal{R}$. Thus, there can be at most one vertex $j \in \mathcal{R}$ on the path such that $\hat{y}\left(F_{j}\right)=0$ in line 4 . Opening $u \in F_{j}$ adds one extra facility to our solution.

Recall that $C$ is the (random) set of all clients within radius $3 R$ from some center in $\mathcal{S}$, where $R$ is the optimal radius. The following two propositions will conclude our analysis.

Proposition 5.13. $|C| \geq t$ with probability one.

Proof. Let $f$ denote the function defined in Algorithm RMCenterRound (i.e., $f(z)=$ $\sum_{j \in V^{\prime}} \sum_{i \in F_{i}} z_{i}$ for any $z \in[0,1]^{V}$.) Using a similar argument as in the proof of Proposition 5.7, one can easily verify that there are at least $f(Y)$ vertices in $V$ that are within radius $3 R$ from some open center in $\mathcal{S}$. Next, it suffices to show that $f(Y) \geq t$.

By definition of $y^{\prime}$ in lines 2 and 3, we have $f\left(y^{\prime}\right) \geq t$ (see the proof of Proposition 5.6.) We now claim that $f\left(y^{\prime}\right)$ is not decreasing after each iteration of the rounding scheme. We check the following cases:

- Case $y^{\prime}$ is rounded by RoundCyCle: observe that $y^{\prime}\left(F_{j}\right)$ is preserved for all $j \in \mathcal{R}$ since $j$ is adjacent to two edges and we increase/decrease the corresponding variables by the same amount. Thus, $f\left(y^{\prime}\right)$ is unchanged.

- Case $y^{\prime}$ is rounded by RoundSinglePath: if $j \in \mathcal{R}$ is not the endpoint of the path, then $j$ is adjacent to two edges on the path and $y^{\prime}\left(F_{j}\right)$ is unchanged. If $j$ is the endpoint, then we increase the variable on the adjacent edge; and hence, $y^{\prime}\left(F_{j}\right)$ will increase. See Figure 3. 
- Case $y^{\prime}$ is rounded by RoundTwoPaths: again, for any $j \in \mathcal{R} \backslash\left\{j_{1}, j_{2}, j_{1}^{\prime}, j_{2}^{\prime}\right\}$, the value of $y^{\prime}\left(F_{j}\right)$ remains unchanged in the process. We now verify the change in $f$ caused by the four endpoints $j_{1}, j_{2}, j_{1}^{\prime}$, and $j_{2}^{\prime}$. Suppose $y_{1}$ is returned, the contribution of these points in $f\left(y_{1}\right)$ is

$$
\begin{aligned}
& c_{j_{1}} y_{1}\left(F_{j_{1}}\right)+c_{j_{2}} y_{1}\left(F_{j_{2}}\right)+c_{j_{1}^{\prime}} y_{1}\left(F_{j_{1}^{\prime}}\right)+c_{j_{2}^{\prime}} y_{1}\left(F_{j_{2}^{\prime}}\right) \\
& =c_{j_{1}}\left(y^{\prime}\left(F_{j_{1}}\right)+\delta_{1}\right)+c_{j_{2}}\left(y^{\prime}\left(F_{j_{2}}\right)-\delta_{1}\right)+c_{j_{1}^{\prime}}\left(y\left(F_{j_{1}^{\prime}}\right)-\delta_{1} \frac{\Delta_{1}}{\Delta_{2}}\right)+c_{j_{2}^{\prime}}\left(y\left(F_{j_{2}^{\prime}}\right)+\delta_{1} \frac{\Delta_{1}}{\Delta_{2}}\right) \\
& =c_{j_{1}} y^{\prime}\left(F_{j_{1}}\right)+c_{j_{2}} y^{\prime}\left(F_{j_{2}}\right)+c_{j_{1}^{\prime}} y^{\prime}\left(F_{j_{1}^{\prime}}\right)+c_{j_{2}^{\prime}} y^{\prime}\left(F_{j_{2}^{\prime}}\right)+\delta_{1}\left(c_{j_{1}}-c_{j_{2}}\right)+\delta_{1} \frac{\Delta_{1}}{\Delta_{2}}\left(c_{j_{2}^{\prime}}-c_{j_{1}^{\prime}}\right) \\
& =c_{j_{1}} y^{\prime}\left(F_{j_{1}}\right)+c_{j_{2}} y^{\prime}\left(F_{j_{2}}\right)+c_{j_{1}^{\prime}} y^{\prime}\left(F_{j_{1}^{\prime}}\right)+c_{j_{2}^{\prime}} y^{\prime}\left(F_{j_{2}^{\prime}}\right) .
\end{aligned}
$$

Hence, $f\left(y_{1}\right)=f\left(y^{\prime}\right)$. Similarly, one can verify that $f\left(y_{2}\right)=f\left(y^{\prime}\right)$.

- Case $y^{\prime}$ is rounded by RoundFinalPath: we have shown in the proof of Proposition 5.12 that $y^{\prime}\left(F_{j}\right)=1$ for all $j \in J$ where $J$ is the set of vertices in $\mathcal{R}$ on the path $\vec{v}$. This fact and the other constraints of $\mathcal{P}_{2}$ ensure that $y^{\prime}\left(F_{j}\right)$ is not decreasing for all $j \in V^{\prime}$.

Proposition 5.14. $\operatorname{Pr}[j \in C] \geq p_{j}$ for all $j \in V$.

Proof. Let $y^{\prime}$ be the vector defined as in lines 2 and 3 of PseudoFRMCEnTERRound. It suffices to show that, for all $j \in V^{\prime}, \operatorname{Pr}\left[Y\left(F_{j}\right)=1\right] \geq y^{\prime}\left(F_{j}\right)$. (Note that $y^{\prime}\left(F_{j}\right) \geq p_{j}$ by constraint (5).) This is because, for any vertex $k \in V \backslash V^{\prime}$, the algorithm RFILTERING guarantees that there exists $j \in V^{\prime}$ such that $F_{k} \cap F_{j} \neq \emptyset$, and $y^{\prime}\left(F_{j}\right)=\sum_{i \in B_{j}} x_{i j} \geq \sum_{i \in V: d(i, k) \leq R} x_{i k}=y^{\prime}\left(F_{k}\right)$. Notice that the event $Y\left(F_{j}\right)=1$ means there is some open center $F_{j}$ and the distance from $k$ to this center should be at most $3 R$. Thus,

$$
\operatorname{Pr}[k \in C] \geq \operatorname{Pr}\left[Y\left(F_{j}\right)=1\right] \geq y^{\prime}\left(F_{j}\right) \geq y^{\prime}\left(F_{k}\right) \geq p_{k}
$$

by constraint (5).

Fix any $j \in V^{\prime}$. Recall that $Y$ is obtained by rounding $y^{\prime}$ and, by Proposition 5.11 and the proof of Proposition 5.12, we have $Y\left(F_{j}\right) \in\{0,1\}$ and $\operatorname{Pr}\left[Y\left(F_{j}\right)=1\right]=\mathrm{E}\left[Y\left(F_{j}\right)\right]$. We now show that the expected value of $y^{\prime}\left(F_{j}\right)$ does not decrease after each iteration of the while-loop.

- Case $y^{\prime}$ is rounded by RoundCyCLE: $y^{\prime}\left(F_{j}\right)$ is unchanged as before.

- Case $y^{\prime}$ is rounded by RoundSinglePath: if $j$ is not the endpoint of $\vec{v}$, then $y^{\prime}\left(F_{j}\right)$ is unchanged. Otherwise, $y^{\prime}\left(F_{j}\right)$ is increased by some $\delta_{1}>0$ with probability one.

- Case $y^{\prime}$ is rounded by RoundTwoPATHs: again, if $j \notin\left\{j_{1}, j_{2}, j_{1}^{\prime}, j_{2}^{\prime}\right\}$, then $y^{\prime}\left(F_{j}\right)$ is unchanged. Now suppose $j=j_{1}$. With probability $\delta_{1} /\left(\delta_{1}+\delta_{2}\right), y^{\prime}\left(F_{j_{1}}\right)$ is increased by $\delta_{2}$, and, with the remaining probability, it is decreased by $\delta_{1}$. Thus, the expected change in $y^{\prime}\left(F_{j_{1}}\right)$ is

$$
\frac{\delta_{1}}{\delta_{1}+\delta_{2}}\left(\delta_{2}\right)+\frac{\delta_{2}}{\delta_{1}+\delta_{2}}\left(-\delta_{1}\right)=0
$$

Similarly, one can verify that the expected values of $y^{\prime}\left(F_{j_{2}}\right), y^{\prime}\left(F_{j_{1}^{\prime}}\right)$, and $y^{\prime}\left(F_{j_{2}^{\prime}}\right)$ remain the same.

- Case $y^{\prime}$ is rounded by RoundFinalPath: we have showed in the proof of Proposition 5.12 that if $j$ is on the path $\vec{v}$, then $Y\left(F_{j}\right)=1$. Otherwise, the constraints of $\mathcal{P}_{2}$ ensure that $Y\left(F_{j}\right)=$ $y^{\prime}\left(F_{j}\right)$.

So far we have proved the following theorem.

Theorem 5.15. PSEUdoFRMCENTERRound will return a random solution $\mathcal{S}$ such that 
$-\mathcal{S}$ is the union of some independent set of $\mathcal{M}$ with (at most) one extra vertex,

$-|C| \geq t$ with probability one, and

$-\operatorname{Pr}[j \in C] \geq p_{j}$ for all $j \in V$.

5.3.3 An Algorithm Satisfying the Matroid Constraint Exactly. Using a similar technique as in Section 4.2.3, we will develop an approximation algorithm for the FRMatCenter problem which always returns a feasible solution. Let $\epsilon>0$ be a small parameter to be determined. Let $\mathcal{U}$ denote the collection of all possible sets of verticies with size at most $\lceil 1 / \epsilon\rceil$ such that $U$ is an independent set of $\mathcal{M}$. Again, we have $|\mathcal{U}| \leq n^{O(1 / \epsilon)}$. Suppose $R$ is the optimal radius to our instance. For any $i \in V$, recall that $\operatorname{RBall}(i, U, R)$ is the set of red vertices within radius $3 R$ from $i$.

Consider the configuration polytope $\mathcal{P}_{\text {config3 }}$ containing points $(x, y, q)$ with the following constraints:

$$
\begin{cases}\sum_{U \in \mathcal{U}} q_{U}=1, & \\ \sum_{i \in B_{j}} x_{i j}^{U} \leq q_{U}, & \forall j \in V, U \in \mathcal{U} \\ \sum_{U \in \mathcal{U}} \sum_{i \in B_{j}} x_{i j}^{U} \geq p_{j}, & \forall j \in V \\ x_{i j}^{U} \leq y_{i}^{U}, & \forall i, j \in V, U \in \mathcal{U} \\ \sum_{i \in W} y_{i}^{U} \leq q_{U} r_{\mathcal{M}}(W), & \forall U \in \mathcal{U}, W \subseteq V \\ \sum_{j \in V} \sum_{i \in B_{j}} x_{i j}^{U} \geq q_{U} t, & \\ y_{i}^{U}=1, & \forall U \in \mathcal{U}, i \in U \\ y_{i}^{U}=0, & \forall U \in \mathcal{U}, i \in V \backslash U,|\operatorname{RBall}(i, U, R)| \geq \epsilon n \\ x_{i j}^{U}, y_{i}^{U}, q_{U} \geq 0, & \forall i, j \in V, U \in \mathcal{U} .\end{cases}
$$

We first claim that $\mathcal{P}_{\text {config } 3}$ is a valid relaxation for the problem.

Proposition 5.16. The polytope $\mathcal{P}_{\text {config3 }}$ is non-empty.

Proof. Suppose $\mathcal{S}$ is a solution drawn from the optimal distribution $\mathcal{D}$. We compute a subset $U_{\mathcal{S}}$ of $\mathcal{S}$ using a similar procedure as in the proof of Proposition 4.4. Recall that $\left|\operatorname{RBall}\left(i, U_{\mathcal{S}}, R\right)\right|<\epsilon n$ for all $i \in \mathcal{S} \backslash U_{\mathcal{S}}$ and $\left|U_{\mathcal{S}}\right| \leq\lceil 1 / \epsilon\rceil$. Since $U_{\mathcal{S}} \subseteq \mathcal{S}, U_{\mathcal{S}}$ is also an independent set of $\mathcal{M}$, implying that $U_{\mathcal{S}} \in \mathcal{U}$.

Now for any $U \in \mathcal{U}$, we set $q_{U}:=\operatorname{Pr}\left[U_{\mathcal{S}}=U\right]$. Let $x_{i j}^{U}$ be the probability of the joint event: $U_{\mathcal{S}}=U$ and $j$ is connected to $i$. Finally, let $y_{i}^{U}$ be the probability of the joint event: $U_{\mathcal{S}}=U$ and $i \in \mathcal{S}$. Then it is clear that $\sum_{U \in \mathcal{U}} q_{U}=1$. Using similar arguments to the proofs of Propositions 4.4 and 4.3 , we have the following inequalities:

$$
\begin{aligned}
\sum_{i \in B_{j}} x_{i j}^{U} & \leq q_{U}, \quad \forall j \in V, U \in \mathcal{U}, \\
\sum_{U \in \mathcal{U}} \sum_{i \in B_{j}} x_{i j}^{U} & \geq p_{j}, \quad \forall j \in V, \\
\sum_{j \in V} \sum_{i \in B_{j}} x_{i j}^{U} & \geq q_{U} t, \\
y_{i}^{U} & =0, \quad \forall U \in \mathcal{U}, i \in V \backslash U,|\operatorname{RBall}(i, U, R)| \geq \epsilon n .
\end{aligned}
$$

Recall that $y_{i}^{U} / q_{U}$ is the $\operatorname{Pr}\left[i \in S \mid U=U_{\mathcal{S}}\right]$. Since $\mathcal{S}$ is independent with probability one, we have $|\mathcal{S} \cap W| \leq r_{\mathcal{M}}(W)$ for all $W \subseteq V$. Therefore,

$$
r_{\mathcal{M}}(W) \geq \mathrm{E}\left[|\mathcal{S} \cap W| \mid U=U_{\mathcal{S}}\right]=\sum_{i \in W} \operatorname{Pr}\left[i \in \mathcal{S} \mid U=U_{\mathcal{S}}\right]=\sum_{i \in W} y_{i}^{U} / q_{U},
$$

for all $W \subseteq V$. 
The other constraints can be verified easily. We conclude that $(x, y, q) \in \mathcal{P}_{\text {config3 }}$.

Next, let us pick any $(x, y, q) \in \mathcal{P}_{\text {config3 }}$ and use the following algorithm to round it.

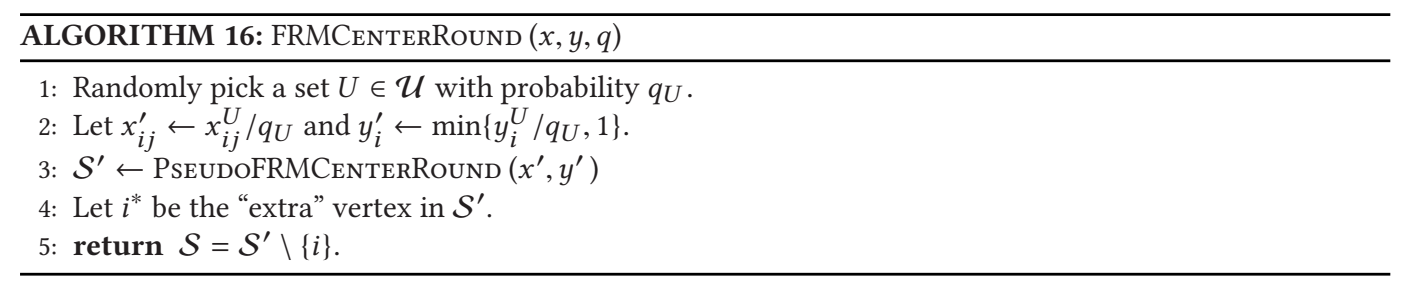

Analysis. We are now ready to prove the second part of Theorem 1.4. Let us fix any $\gamma>0$ and set $\epsilon:=\gamma^{2}$. Also, let $\mathcal{E}(U)$ be the event that Algorithm 16 selects $U \in \mathcal{U}$. Note that $\left(x^{\prime}, y^{\prime}\right)$ satisfies the following inequalities:

$$
\begin{aligned}
\sum_{j \in V} \sum_{i \in B_{j}} x_{i j}^{\prime} & \geq t, \\
\sum_{i \in B_{j}} x_{i j}^{\prime} & \leq 1, \quad \forall j \in V, \\
\sum_{i \in B_{j}} x_{i j}^{\prime} & =\sum_{i \in B_{j}} x_{i j} / q_{U}, \quad \forall j \in V, \\
x_{i j}^{\prime} & \leq y_{i}^{\prime}, \quad \forall i, j \in V, \\
\sum_{i \in W} y_{i}^{\prime} & \leq r_{\mathcal{M}}(W), \quad \forall W \subseteq V .
\end{aligned}
$$

Moreover, every $i \in U$ satisfies $y_{i}^{\prime}=1$; every $i \in V \backslash U$ with $\operatorname{RBall}(i, U, R) \geq \epsilon n$ satisfies $y_{i}^{\prime}=0$.

Recall that the algorithm PseudoFRMCEnTerRound will return a solution $\mathcal{S}^{\prime}$, which is the union of a basis of $\mathcal{M}$ with an extra center $i^{*}$. The vertex $i^{*}$ has $y_{i^{*}} \in(0,1)$, and in particular $i^{*} \notin U$. By removing $i^{*}$ from $\mathcal{S}^{\prime}$, we ensure that the resulting set is a basis of $\mathcal{M}$ with probability one.

Now we shall prove the coverage guarantee. By Theorem $5.15, \mathcal{S}^{\prime}$ covers at least $t$ vertices within radius $3 R$. Any blue vertex can be connected to some center in $U$ and hence is not affected by the removal of $i^{*}$. Because $i^{*}$ covers at most $\epsilon n$ other red vertices, we have

$$
|C| \geq t-\epsilon n=t-\gamma^{2} n .
$$

For any $j \in V$, let $X_{j}$ be the indicator random variable for the event that $d\left(j, \mathcal{S}^{\prime}\right) \leq 3 R$ but $d\left(j, \mathcal{S}^{\prime} \backslash\left\{i^{*}\right\}\right)>3 R$. We say that $j$ is a bad vertex iff $\mathrm{E}\left[X_{j}\right] \geq \gamma$, otherwise, $j$ is good. Again, $\sum_{j \in V} X_{j} \leq \epsilon n$ with probability one. Thus, there can be at most $\epsilon n / \gamma$ bad vertices. Letting $T$ be the set of good vertices, we have

$$
|T| \geq n-\epsilon n / \gamma=(1-\gamma) n
$$

By Theorem $5.15, \operatorname{Pr}\left[j\right.$ is covered by $\left.\mathcal{S}^{\prime}\right] \geq p_{j}$. So, for any $j \in T$, we have

$$
\operatorname{Pr}[j \in C] \geq \operatorname{Pr}\left[j \text { is covered by } \mathcal{S}^{\prime}\right]-\operatorname{Pr}\left[X_{j}=1\right] \geq p_{j}-\gamma .
$$

\section{ACKNOWLEDGMENTS}

We thank the APPROX 2017 and TALG reviewers for their helpful suggestions to improve the presentation of this article. 


\section{REFERENCES}

[1] Sara Ahmadian, Ashkan Norouzi-Fard, Ola Svensson, and Justin Ward. 2016. Better guarantees for $k$-means and Euclidean $k$-median by primal-dual algorithms. CoRR abs/1612.07925. http://arxiv.org/abs/1612.07925

[2] Jaroslaw Byrka, Thomas Pensyl, Bartosz Rybicki, Aravind Srinivasan, and Khoa Trinh. 2015. An improved approximation for $k$-median, and positive correlation in budgeted optimization. In Proceedings of the Annual ACM-SIAM Symposium on Discrete Algorithms (SODA'15). 737-756.

[3] Deeparnab Chakrabarty, Prachi Goyal, and Ravishankar Krishnaswamy. 2016. The non-uniform k-center problem. In Proceedings of the 43rd International Colloquium on Automata, Languages, and Programming (ICALP'16), Vol. 55. 67:1-67:15.

[4] Moses Charikar, Samir Khuller, David M. Mount, and Giri Narasimhan. 2001. Algorithms for facility location problems with outliers. In Proceedings of the 12th Annual ACM-SIAM Symposium on Discrete Algorithms (SODA'01). Society for Industrial and Applied Mathematics, Philadelphia, PA, 642-651. http://dl.acm.org/citation.cfm?id=365411.365555

[5] Danny Z. Chen, Jian Li, Hongyu Liang, and Haitao Wang. 2013. Matroid and knapsack center problems. Proceedings of the 16th International Conference on Integer Programming and Combinatorial Optimization (IPCO'13), 110-122. DOI : https://doi.org/10.1007/978-3-642-36694-9_10

[6] I. M. Gel'fand and V. V. Serganova. 1987. Combinatorial geometries and torus strata on homogeneous compact manifolds. Russian Mathematical Surveys 42, 2 (1987), 133. http://stacks.iop.org/0036-0279/42/i=2/a=R03

[7] David G. Harris, Thomas Pensyl, Aravind Srinivasan, and Khoa Trinh. 2017. Symmetric randomized dependent rounding. CoRR abs/1709.06995.

[8] Dorit S. Hochbaum and David B. Shmoys. 1986. A unified approach to approximation algorithms for bottleneck problems. Fournal of the ACM 33, 3 (May 1986), 533-550. DOI: https://doi.org/10.1145/5925.5933

[9] Wen-Lian Hsu and George L. Nemhauser. 1979. Easy and hard bottleneck location problems. Discrete Applied Mathematics 1, 3 (1979), 209-215. DOI : https://doi.org/10.1016/0166-218X(79)90044-1

[10] Tapas Kanungo, David M. Mount, Nathan S. Netanyahu, Christine D. Piatko, Ruth Silverman, and Angela Y. Wu. 2004. A local search approximation algorithm for $k$-means clustering. Computational Geometry 28, 2-3 (2004), 89112. DOI : https://doi.org/10.1016/j.comgeo.2004.03.003

[11] Ravishankar Krishnaswamy, Amit Kumar, Viswanath Nagarajan, Yogish Sabharwal, and Barna Saha. 2011. The matroid median problem. In Proceedings of the Annual ACM-SIAM Symposium on Discrete Algorithms (SODA'11). SIAM, 1117-1130.

[12] Lap-Chi Lau, R. Ravi, and Mohit Singh. 2011. Iterative Methods in Combinatorial Optimization (1st ed.). Cambridge University Press, New York, NY.

[13] Shi Li and Ola Svensson. 2013. Approximating $k$-median via pseudo-approximation. In Proceedings of STOC. 901-910.

[14] Alexander Schrijver. 2003. Combinatorial Optimization: Polyhedra and Efficiency. Vol. 24. Springer Science \& Business Media.

[15] Chaitanya Swamy. 2014. Improved approximation algorithms for matroid and knapsack median problems and applications. In APPROX/RANDOM 2014, Vol. 28. 403-418.

[16] David P. Williamson and David B. Shmoys. 2011. The Design of Approximation Algorithms. Cambridge University Press. I-XI, 1-504 pages.

Received October 2017; revised December 2018; accepted February 2019 